\title{
Befruchtung und Embryobildung bei Oenothera Lamarckiana und einigen verwandten Arten.
}

\author{
Von 0. Renner.
}

(Mit Tafel XII u. XIII und 15 Abbildungen im Text.)

\section{Die zytologischen Vorgänge bei einigen Artkreuzungen.}

Auf Veranlassung von Herrn Professor R. Goldschmidt habe ich im Sommer 1912 Kreuzungen zwischen Oenothera biennis, muricata und Lamarckiana hergestellt und die Fruchtknoten in verschiedenen Stadien für zytologische Untersuchung fixiert. Die Flemming'schen Gemische erwiesen sich als ganz unbrauchbar, gute Fixierung wurde dagegen meistens erreicht mit der von Petrunkewitsch ${ }^{1}$ ) modifizierten Flüssigkeit nach Gilson. Gefärbt wurde mit Eisenhämatoxylin, mitunter wurde zur Gegenfärbung Eosin verwendet.

Die Rhaphiden, die in allen Teilen der Fruchtknotenwand der Önotheren in großen Mengen vorkommen, werden von der Gils o n'schen Flüssigkeit nicht aufgelöst. Auf ihre nachträgliche Beseitigung mittels Salzsäure wurde verzichtet. Sie richten in den Schnitten freilich häufig Schaden an, am wenigsten noch, wenn man die.Fruchtknoten längs schneidet und dabei das Messer von einer Schnittfläche des Fruchtknotenstückes zur anderen führt, nicht von einer Flanke zur anderen. Einigermaßen herangewachsene Samenanlagen wurden vor oder nach der Fixierung aus dem Fruchtknoten herausgenommen und so ohne die gefährlichen Gewebe eingebettet und geschnitten.

Oenothera biennis und Lamarckiana. waren in kräftigen Gartenexemplaren unbekannter Herkunft vorhanden. Für Übersendung lebenden Materials von O. muricata bin ich Herrn Geheimrat Oltmanns und Herrn Dr. Tröndle in Freiburg i. Br., Herrn Professor G. Mahler

1) Zoolog. Jahrb., Abt. f. Morph., 1901, Bd. XIV, pag. 576. Die Zusammensetzung ist: $40 \%$ iger Alkohol $500 \mathrm{ccm}$, Eisessig $90 \mathrm{ccm}$, reine Salpetersäure $10 \mathrm{ccm}$, Sublimat bis zur Sättigung. Fixiert wird höchstens 12 Stunden, ausgewaschen wird mit Jodalkohol.

Flora, Bd. 107. 
in Ulm und Herrn Dr. Pöverlein in Ludwigshafen zu großem Dank verpflichtet. Die mit dem Pollen solcher süddeutschen Pflanzen bestäubten Fruchtknoten von O. biennis sind von Professor Goldschmidt untersucht worden, und das Ergebnis der Untersuchung ist bereits veröffentlicht (1912). Später kamen Pflanzen von O. muricata im Münchener Garten zur Blüte, die Herr Geheimrat v. Goebel vom Lido bei Venedig mitgebracht hatte und die in der Blattfarbe und in der Behaarung nicht ganz mit den bei uns eingeschleppten Formen der O. muricata übereinstimmen. Deren Pollen wurde weiterhin zur Bestäubung von O. biennis verwendet, und an denselben Pflanzen wurde auch die reziproke Kreuzung mit dem Pollen von O. biennis vorgenommen. Bestäubt wurde in Pergamentbeuteln, einen Tag nach der Kastrierung.

Nach den von de Vries (1911) ermittelten Vererbungsverhältnissen vermutete Goldschmidt, daß bei den Kreuzungen zwischen Oenothera biennis und muricata im befruchteten Ei nur der Spermakern sich entwickele, während der Eikern zugrunde gehe. Die zytologische Untersuchung der Kreuzung 0 . biennis $\$ \times$ muricata $\sigma^{\top}$ schien das zu bestätigen, besonders insofern, als die Kerne des jungen Bastardembryo die haploide Chromosomenzahl 7 zeigen sollten, nicht die diploide Zahl 14. Das Ergebnis war also ein anderes als das, welches Strasburger an dem ebenfalls streng patroklinen Bastard Fragaria virginiana $\times$ elatior erhalten hat; hier soll normale Befruchtung, keine Merogonie vorliegen (1909, pag. 41).

Eine Nachprüfung seiner Resultate, vor allem auch die Aufsuchung der ersten Mitosen im befruchteten Ei, hielt Herr Professor Goldschmidt selber für wünschenswert. Selbstverständlich wollte ich zugleich mit dieser Nachuntersuchung auch die entsprechenden Verhältnisse beim reziproken Bastard 0 . muricata $Q \times$ biennis $\sigma^{x}$, sowie bei den Kreuzungen zwischen 0 . Lamarckiana einerseits und 0 . biennis und muricata andererseits studieren, weil das züchterische Verhalten dieser Bastarde nach de Vries dasselbe ist wie das der Bastarde zwischen o. biennis und muricata. Was die Untersuchung dieser Kreuzungen ergab, wird im folgenden mitgeteilt.

Herr Professor V. Grégoire hatte die große Liebenswürdigkeit, auf die Bitte von Herrn Professor Goldschmidt hin alle wichtigen Präparate sorgfältig zu studieren, und ich möchte ihm dafür auch hier meinen verbindlichsten Dank aussprechen. Er ist in allen Fällen zu dem gleichen Ergebnis gekommen wie ich. Das Hauptresultat habe ich, vor seiner Antwort, in den Berichten der Deutschen Botanischen Gesellschaft (1913, pag. 334) veröffentlicht. 
Herr Dr. J. Seiler war so freundlich, für Herrn Professor Goldschmidt einige Schnittserien herzustellen, in denen sich wichtige Teilungsstadien fanden. Auch ihm danke ich bestens.

a) Die somatischen Chromosomenzahlen der verwendeten Materialien.

Wurden Oenothera biennis und O. muricata-Venedig mit arteigenem Pollen bestäubt, so zeigten die Embryonen 14 Chromosomen, wie Goldschmidt schon mitgeteilt hat. Eine Äquatorialplatte von O. muricata gibt die Textfig. $1 b$ wieder. Dieselbe Chromosomenzahl findet sich bei O. muricata auch in den meisten Endospermzellen (Metaphase, Textfig. 1c). Das spricht für Befruchtung des sekundären Embryosackkerns; denn dieser ist haploid, nicht diploid, weil Antipodenbildung und Polkernfusion bei Oenothera unterbleiben (vgl. unten). Nicht selten
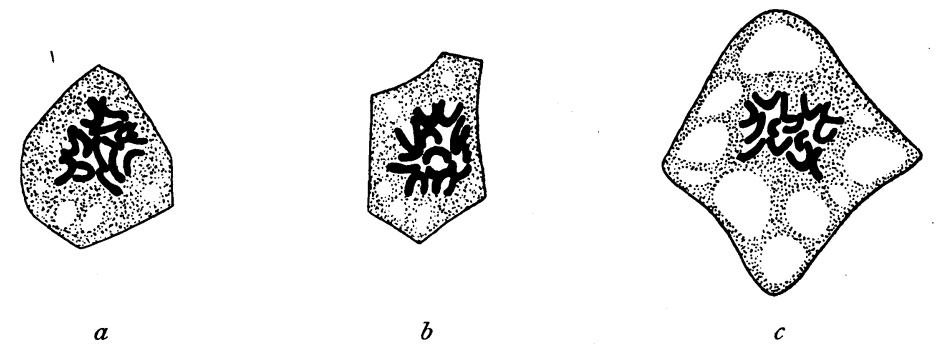

Fig. 1. $a$ O. biennis, Äquatorialplatte aus dem Nucellus. $b$ und $c$ o. biennis $\times$ muricata; $b$ Kern aus dem Embryo, $c$ aus dem Endosperm. Zeiss Apochr. $2 \mathrm{~mm}$, Komp.-Ok. 18; auf $3 / 4$ verkl.

verschmelzen in späteren Stadien, wie auch sonst in älteren Endospermen, mehrere Kerne miteinander, und dann sieht man in den Mitosen eine große Zahl von Chromosomen auftreten.

Auch im Nucellus der Samenanlagen der O. biennis wurden Mitosen mit 14 Chromosomen gefunden (Textfig. 1a).

Bei O. muricata-Süddeutschland und bei den zur Kreuzung verwendeten Individuen von O. Lamarckiana wurde die Feststellung der Chromosomenzahl versäumt, doch ist kaum zu zweifeln, daß sie auch hier 14 betrug.

Einen Unterschied in der Größe und Form der Chromosomen habe ich zwischen 0 . biennis und muricata nicht entdecken können (vgl. Goldschmidt, pag. 342). 
b) Die Chromosomenzahl in Keimlingen und vielzelligen Embryonen der Kreuzung O. biennis $\times$ muricata.

Gut ausgebildete Samen des Bastardes 0 . biennis $\times$ muricataSüddeutschland, die im Herbst 1912 geerntet worden waren und Anfang April 1913 auf Fließpapier ausgelegt wurden, keimten zu einem kleinen Teil nach wenigen Tagen. Die Keimwurzeln wurden teils nach Gils on, teils nach Fle m ming fixiert und quer geschnitten. Prophasen, Äquatorialplatten in Flächenansicht und Anaphasen in Polansicht wurden in großer Zahl beobachtet. Die Chromosomenzahl ist auch an den Äquatorialplatten oft nicht mit vollkommener Sicherheit festzustellen, weil die Chromosomen meistens dicht beisammen und teilweise übereinander
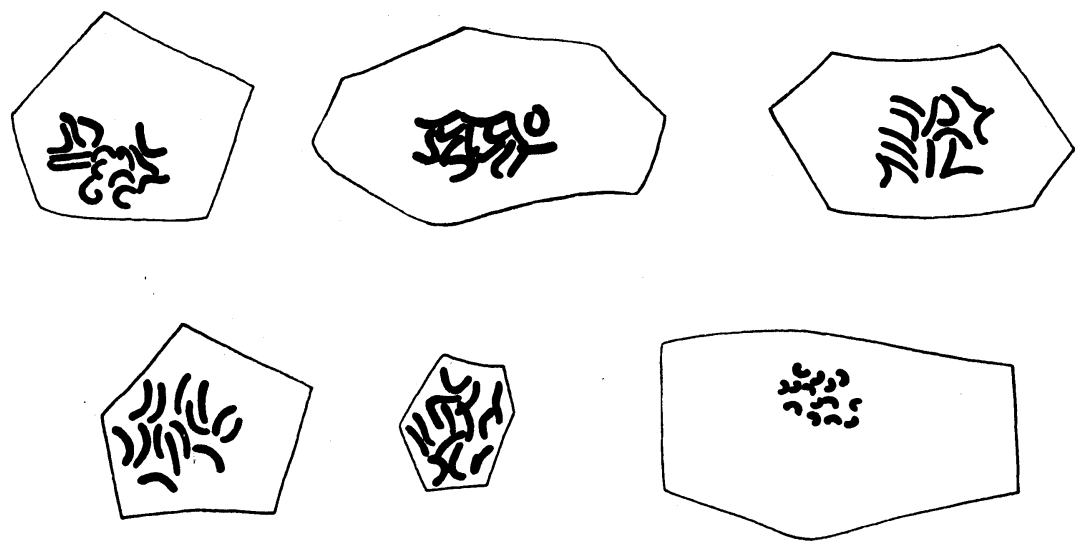

Fig. 2. O. biennis $\times$ muricata, fünf Äquatorialplatten und eine Tochterplatte aus verschiedenen Geweben der Wurzelspitze. Apochr. $2 \mathrm{~mm}$, Ok. 18; auf $3 / 4$ verkl.

liegen. Doch konnte vielfach bestimmt die Zahl 14 ermittelt werden (vgl. die Textfig. 2), und bei keinem einzigen Kern war die Vermutung möglich, daß die Chromosomenzahl sich von 14 weit nach unten oder nach oben entfernen könnte.

Bei der Nachprüfung der Goldschmidt'schen Präparate, die mir vom Autor zur Verfügung gestellt wurden, kam ich ebenfalls zu dem Ergebnis, daß in solchen Embryonen des Bastards O. biennis $\times$ muricata, die schon die Ausgliederung der Kotyledonen zeigen oder auch noch auf dem Stadium der Keimkugel stehen, nur diploide Kerne vorkommen. Goldschmidt gibt selber an, daß er in den Äquatorialplatten meistens 14 Chromosomen gesehen hat (pag. 338), und ich habe überhaupt nie mit Sicherheit 7 Chromosomen zählen können. Von den Vorbildern zu den hierhergehörigen Figuren Goldschmidt's (3 und 5) 
habe ich die zu $3 c, 3 d, 3 e, 5$ links in seinen Präparaten wiedergefunden und als diploid erkannt. Die Fig. 1 und 2 der Taf. XII geben eine Äquatorialplatte und eine Prophase aus einem Golsdchmidt'schen Präparat wieder. Auch im Endosperm solcher junger Samen habe ich wiederholt mit Sicherheit 14 Chromosomen gezählt (Taf. XIII, Fig. 12 Äquatorialplatte, Fig. 13 Tochterplatte). Die Deutung, die Goldschmidt seiner Fig. $3 c$ gibt, daß nämlich nach der Spaltung die Tochterchrọmosomen paarweise lange nebeneinander liegen bleiben, hat nicht viel Wahrscheinlichkeit; die gepaarten Chromosomen müßten in diesem Fall, worauf auch Herr Prøfessor Grégoire hingewiesen hat, ja übereinander liegen, nicht alle in einer Ebene nebeneinander, wie die Figur es zeigt.

Wie Goldschmidt (pag. 340) hervorhebt, müssen wir mit der Möglichkeit einer Wiederherstellung der diploiden Chromosomenzahl rechnen, auch wenn der Bastardembryo sein Dasein mit der haploiden Zahl beginnt. Und die haploide Zahl kann entweder dadurch zustande kommen, daß eine Kernverschmelzung ganz unterbleibt, oder durch nachträgliche Ausstoßung eines Chromosomensatzes. Es mußte also der Vorgang der Befruchtung und die früheste Embryoentwicklung so vollständig wie möglich verfolgt werden.

c) Der Embryosack der Önotheren vor und kurz nach der Befruchtung.

Im empfängnisreifen Embryosack von O. biennis (Textfig. 3, und ebenso bei den anderen Arten) ragt die Eizelle über die Synergiden vor. Sie besitzt sehr wenig Plasma, und der Kern, der einen Nucleolus besitzt, liegt am Scheitel, dem Embryosackraum zugekehrt. Die Synergiden sind reich an dichtem Plasma, das zur Hauptsache in der Richtung gegen die Mikropyle hin zusammengedrängt ist und den einnucleoligen Kern einschließt, während das gegen den Embryosack gekehrte Ende von einer großen Vakuole eingenommen ist. Der sekundäre Embryosackkern, ebenfalls mit einem Nucleolus (er entspricht einem Polkern der normalen, achtkernigen Embryosäcke), sitzt dem Ei meistens dicht auf; gelegentlich ist er durch einen Plasmastrang vom Ei getrennt, nie berührt er eine Synergide. Antipoden und unterer Polkern fehlen ganz ${ }^{1}$ ).

Wenn der weite, dickwandige Pollenschlauch den dicken Nucellus durchwandert hat und beim Embryosack ankommt, sind die Synergiden nicht, wie Geerts (pag. 143) angibt, schon zerstört, sondern wie in anderen Fällen ergießt der Pollenschlauch seinen Inhalt in eine der bis dahin

1) Über die Entwicklungsgeschichte vgl. Modilewski u. Geerts. 
unversehrten Synergiden, als eine eigentümlich homogene, stark färbbare, von noch stärker farbspeichernden Klumpen durchsetzte Masse, die nur langsam an Volumen abnimmt und zuletzt, wenn die Embryobildung schon weit vorgeschritten ist, verschwindet (Textfig. 4a). Auch die zweite Synergide, die vom Pollenschlauch nicht unmittelbar zerstört wird, fällt bald zusammen und verschwindet rasch (Textfig. $4 a, b, c$ ).

Die generativen Kerne konnten, einige unsichere Fälle ausgenommen, weder im Pollenschlauch noch im Embryosack beobachtet werden; die ersten Stadien nach der Bestäubung waren leider nur mit Flemming fixiert worden und unbrauchbar. Der sekundäre Embryosackkern scheint sich sehr bald nach der Befruchtung zu teilen. Er verläßt seinen

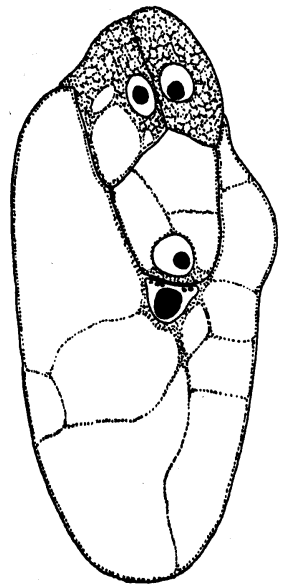

$a$

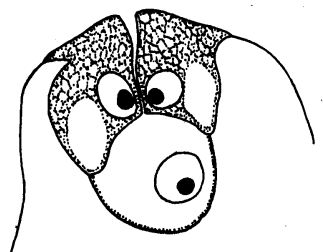

$b$

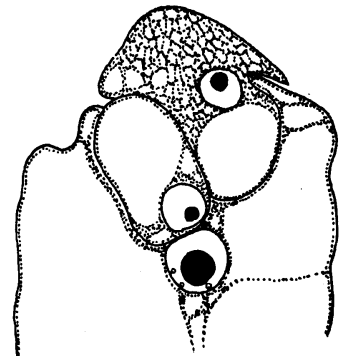

$c$

Fig. 3. 0. biennis. a Embryosack mit zwei Synergiden, Ei und Embryosackkern. $b$ zwei Synergiden und Ei. $c$ Ei, Embryosackkern und eine Synergide. Apochr. $2 \mathrm{~mm}, 0 \mathrm{k}$. 6 ; auf $2 / \mathrm{s}$ verkl.

Platz auf dem Scheitel des Eies, und die meist zweinukeoligen Endospermkerne besetzen unter regelmäßig simultaner Teilung rasch die ganze Embryosackwand bis zum Chalazaende. Das befruchtete Ei, in dessen Kern meist zwei Nukleolen sichtbar sind, bleibt währenddessen geraume Zeit unverändert. Erst wenn schon eine größere Zahl von Endospermkernen vorhanden ist, fängt das $\mathrm{Ei}$ an zu wachsen. Die rundliche Blase treibt am Scheitel eine ziemlich schmale Ausstülpung und wird dadurch flaschenförmig (Textfig. 4c); der Kern und die Hauptmasse des Plasmas halten sich im engen Spitzenteil. Jetzt tritt der Eikern in Teilung; die Spindelachse fällt mit der Achse der Flasche zusammen (Textfig. $4 d$ ), die Teilungswand wird quer gelegt und trennt einen dünnen 
Kopf vom meist aufgeblasenen Fußteil (Textfig. 4e). Nicht selten unterbleibt die Bildung der Wand nach der ersten Kernteilung (Textfig. 4f). Die nächste Teilung im Kopf halbiert diesen meist der Länge nach, er wird also zum eigentlichen Embryo und zerlegt sich auch bald durch zwei gleichzeitige Querteilungen in Quadranten. Der zunächst einzellige blasenförmige Suspensor trägt seinen Kern an der Spitze, dicht beim Embryo. Er teilt sich in langen Zwischenräumen der Quere nach und kann an Embryonen, die schon zweilappig sind, einen Faden von 3-4 Zellen darstellen.
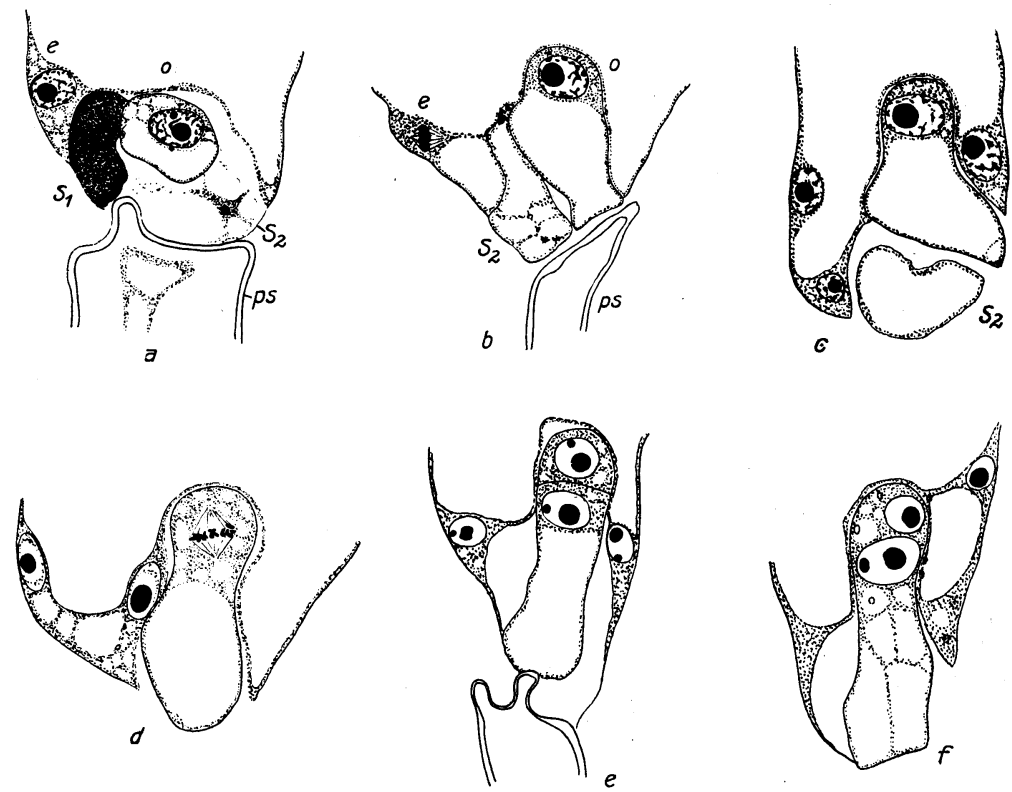

Fig. 4. 0 . biennis $\times$ muricata. $a-c$ befruchtete Eier. $d$ befruchtetes Ei mit der ersten Spindel. $e$ zweizelliger Embryo. $f$ zweikerniger Embryo. o bezeichnet Ei, $s_{1}$ die vom Pollenschlauch angebohrte, $s_{2}$ die andere Synergide, $e$ Endospermkern, ps Pollenschlauch. Apochr. $2 \mathrm{~mm}, \mathrm{Ok} .6$; auf $2 / 3$ verkl.

Während der Embryo in normaler Weise heranwächst, vermehren sich auch die Endospermkerne im Plasmabelag des sich stetig vergrößernden Embryosackes, aber nicht überall gleichmäßig (Textfig. 5). Die Endospermkerne liegen nämlich besonders dicht um den Suspensor herum und verbreiten sich von hier aus auch über den jungen Embryo, dem sie dicht anliegen, und die größte Endospermmasse bildet sich in dem engen Chalazaende des Embryosackes. Hier ist an Stelle des dünnen Plasmabelages eine vollständige Plasmafüllung vorhanden, die 
zahlreiche große Kerne einschließt. Die Teilungsfiguren sind kleiner als im Embryo, die Chromosomen dicht zusammengedrängt und schwer zu zählen. In Samen, die schon zweilappige Embryonen enthalten, sind mehrere Schichten von großen Endospermzellen mit Membran vorhanden; die Kerne sind sehr groß und enthalten viel Chromatin, sie teilen sich nicht mehr alle gleichzeitig wie im Anfang der Endospermbildung.

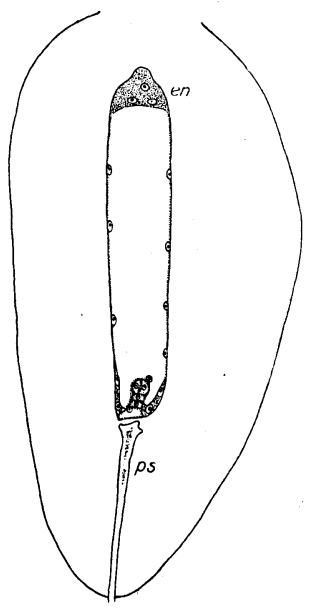

Fig. 5. 0. biennis $\times$ muricata. Nucellus einer befruchteten Samenanlage, mit Pollenschlauch, mehrzelligem Embryo und zahlreichen Endospermkernen. Bald füllt sich der ganze Embryosackraum um den Embryo herum mit zartwandigem Endosperm, das vom reifenden Embryo bis auf eine sehr dünne Schicht wieder aufgezehrt wird.

d) Die Embryobildung bei der Kreuzung 0 . biennis $O \times$ muricata $O^{\top}$.

Die Figuren, die die obige Schilderung von der Entwicklung des jungen Embryo und des Endosperms illustrieren, sind zur Hauptsache nach Präparaten der Kreuzung 0 . biennis $\times$ muricata-Venedig entworfen; die Kreuzung $O$. biennis $\times$ muricata-Süddeutschland, die mir in den Goldschmidt'schen Präparaten vorliegt, verhält sich genau gleich. Es ist also zunächst hervorzuheben, daß, entgegen der Angabe Goldschmidt's, die Befruchtung normal verläuft. Das frisch befruchtete Ei enthält einen einzigen Kern mit zwei Nukleolen, der zweifellos durch Verschmelzung des Eikernes mit dem Spermakern entstanden ist. In vielen Dutzenden von Eiern dieses Stadiums waren nie zwei getrennte Vorkerne zu sehen, von denen der eine dann zugrunde gehen könnte. Was Goldschmidt in seiner Fig. $1 a$ abbildet, ist ein zweikerniger Embryo, in dem nach der Kernteilung keine Querwand gebildet wurde (wie in Textfig. 4f). Über die Altersbestimmung kann deswegen nie ein Zweifel bestehen, weil neben dem frisch befruchteten Ei regelmäßig die noch gut erhaltenen Reste der Synergiden zu finden sind, die im zweizelligen bzw. zweikernigen Zustand des Embryo schon fehlen.

Die erste Teilung im befruchteten Ei kam in mehreren frühen Prophasen (davon eine in Taf. XII, Fig. 3 wiedergegeben), in einer späten Prophase (Taf. XII, Fig. 4) und in zwei Anaphasen (Taf. XII, Fig. 5 und 7) zur Beobachtung. Die Mitose ist typischer Art, und die Chromosomenzahl ist ohne jeden Zweifel die diploide. Ob später, etwa in der Anaphase, Chromosomen ausgestoßen werden oder nicht, konnte wegen des Mangels 
der betreffenden Stadien nicht direkt entschieden werden. Doch läßt die zweite Mitose im Embryo keinen $Z_{w}$ weifel darüber, daß eine Ausstoßung von Chromatin unterbleibt. Der zweite Teilungsschritt wurde, besonders in Prophasen verschiedenen Alters, ziemlich häufig beobachtet, sowohl in zweizelligen Embryonen in der Kopfzelle als in mehrzelligen Embryonen in der sich später teilenden Fußzelle, und immer sind die Chromosomen in der diploiden Zahl vorhanden (Taf. XII, Fig. 6 u. 8). Die Prophase der dritten Teilung wurde in einem nachgefärbten Goldschmidt'schen Präparat gefunden; sie unterscheidet sich nicht von den vorhergehenden Mitosen. Embryonen mit 12-20 Zellen lagen nur in Flemming-Mąterial von 0 . biennis $\times$ muricata-Süddeutschland vor; in den Prophasen sind die Chromosomen infolge der schlechten Fixierung zu kleinen rundlichen Klümpchen zusammengezogen, nicht wurmförmig, aber die Zahl dieser Klümpchen läßt sich oft mit Sicherheit zu 14 bestimmen (Taf. XII, Fig. 11). Über die noch älteren Embryonen ist oben schon berichtet. Im Sommer 1913 wurde nochmals O. biennis mit dem Pollen von O. muricata aus Freiburg i. Br. bestäubt. Die Embryonen wurden als Kugeln mit 8-12 Zellen im Durchmesser untersucht und zeigten diploide Kerne.

Von Goldschmidt's Figuren bezieht sich Fig. 2 auf die zweite Mitose (im Suspensor). Die linke, zerschnittene Tochterplatte schien mir lange haploid zu sein, nach sorgfältiger Differenzierung, die Herr Dr. Seiler vorgenommen hat, ließ sie aber etwa ein Dutzend Chromosomen erkennen; die unzerschnittene Tochterplatte blieb unanalysierbar. Herr Professor Grégoire schließt sich meiner Zählung an.

In einem zweikernigen Embryo hat Goldschmidt den einen Kern scheinbar im Zustand der Auflösung gefunden (Fig. 1b, pag. 335). Ähnliche Bilder sind mir auch bei 0 . biennis $\times$ muricata-Venedig zu Gesicht gekommen, aber hier sind beide Kerne in dem abnormalen Zustand (Taf. XII, Fig. 9 und 10): die Kernmembran ist undeutlich, und die Nukleolen haben sehr unregelmäßige Gestalt. Solche Zustände von Kernen finden sich auch im Endosperm. Ob es sich wirklich um degenerierende Kerne handelt oder nur um ungewöhnliche, vorübergehende Ruhestadien, möchte ich nicht entscheiden ${ }^{1}$ ). Keinesfalls aber scheint es mir möglich, in den fraglichen Bildern einen Hinweis darauf zu erblicken, daß aus der Zygote das mütterliche Kernelement entfernt wird.

1) Herr Prof. Grégoire hält es ebenfalls für sehr fraglich, ob hier eine Degeneration vorliegt. 
Auch im Endosperm ist die Chromosomenzahl von Anfang an die diploide (vgl. Taf. XIII, Fig. 14, 15, 16 aus sehr jungem Endosperm; Fig. 12 und 13 aus älteren Samen mit schon septiertem Endosperm).

e) O. muricata-Venedig $\bigcirc \times$ biennis $0^{x}$.

Kurze Zeit nach der Befruchtung, wenn die ziemlich normal aussehenden Embryonen 3-4-zellig sind, ist das Endosperm noch sehr weit zurück (Textfig. $6 b-d$ ). Es besteht im höchsten Fall aus vier Kernen, häufig sind nur zwei Kerne vorhanden, mitunter ist der Kern, der dem Endosperm den Ursprung geben sollte, überhaupt nicht geteilt und liegt in nächster Nähe des Embryo. Sind Kernteilungep eingetreten, so finden sich die Kerne teilweise in beträchtlicher Entfernung vom Embryo,
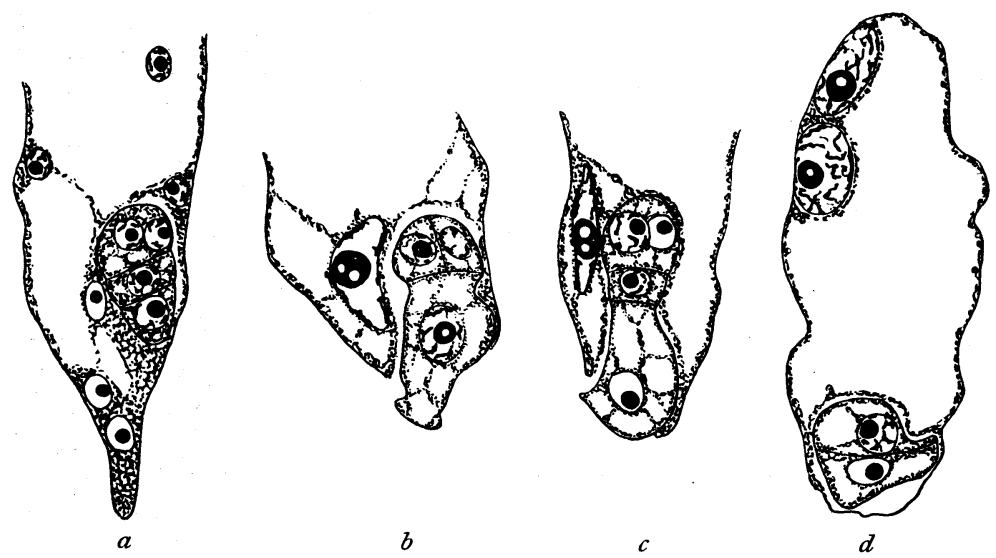

Fig. 6. $a$ 0. muricata $\times$ muricata (?). $b-d \quad$ o. muricata $\times$ biennis. Junge Embryonen mit Endospermkernen. Apochr. $2 \mathrm{~mm}$, Ok. 6; auf $1 / 2$ verkl.

gegen den Chalazapol des Embryosackes hin, wie bei normaler Endospermbildung. Noch auffallender als die geringe Zahl der Endospermkerne ist ihre mächtige Größe; sie übertreffen die normalen an Volumen um das Vielfache und besitzen sehr große, vakuolige Nukleolen. Zum Vergleich mag der normale Embryosack (Textfig. 6a) dienen, aus dem gleichen Fruchtknoten wie die in Textfig. $6 b-d$ wiedergegebenen Embryosäcke, also genau so alt wie diese; von den sehr zahlreichen, kleinen Endospermkernen, die der Embryosack auf seinem ganzen Umfang, vom Embryo bis zum Chalazapol, enthält, sind nur ganz wenige in dem gezeichneten Stück eines Schnittes getroffen. Der Embryo ist in seinem Kopfteil schief getroffen, er besteht ebenfalls aus mehr Zellen als die Embryonen der abnormalen Embryosäcke; die Zellen scheinen auch etwas mehr Plasma zu enthalten. 
Von solchen gesunden Embryosäcken, mit normalem Embryo und Endosperm, wurden in einem Fruchtknoten zwei Stück neben vielen kranken gefunden, während zahlreiche andere Serien nur kranke Embryosäcke enthalten; wahrscheinlich verdanken also die gesunden Embryonen einer Befruchtung der O. muricata mit dem eigenen Pollen die Entstehung. Die Antheren der Venediger Form öffnen sich sehr früh in der Knospe und so mögen in einer Blüte vor der Kastrierung einige Pollenkörner auf die Narbe gekommen sein. Die mutmaßliche nicht beabsichtigte Verunreinigung hat aber das Gute, daß an der normalen Beschaffenheit der Samenanlagen nicht gezweifelt werden kann. Wenn die Embryonen und Endosperme der Kreuzung muricata-Venedig $\times$ biennis

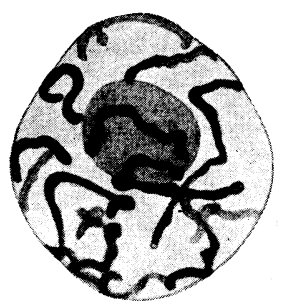

Fig. 7.

Fig. 7. O. muricata $\times$ biennis. Größeres Stück einer Prophase aus einem dreizelligen Embryo; der nächste Schnitt enthält noch zahlreiche Fragmente von Chromosomen. Apochr. $2 \mathrm{~mm}$, Ok. 18.

Fig. 8. 0. muricata $\times$ biennis. Längsschnitt durch einen jungen Samen, mehrere

Wochen nach der Bestäubung.

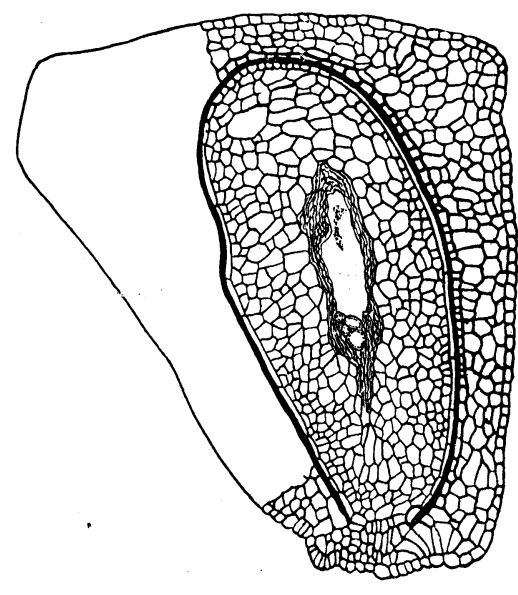

Fig. 8.

sich nicht normal entwickeln, so beruht das also auf einer Unverträglichkeit zwischen den Embryosäcken der O. muricata und den Spermakernen der 0 . biennis, und diese Unverträglichkeit muß wohl genotypisch bedingt sein.

Mitosen wurden unter einer großen Zahl von kranken Samenanlagen in einem einzigen, dreizelligen Embryo gefunden. Die beiden Kerne des längsgeteilten Kopfes befinden sich in der Vorbereitung zur Teilung, der eine in früher Prophase, mit langen, gewundenen Chromosomen (Textfig. 7), der andere in später Metaphase, die leider von der Seite zu sehen ist. Eine genaue Zählung der Chromosomen ist nicht möglich, doch ist die Zahl zweifellos ungefähr ein Dutzend. Demnach muß auch bei dieser Kreuzung normale Befruchtung eintreten. Im Endosperm wurde leider kein einziger Kern gefunden, der eine Schätzung der Chromosomenzahl erlaubt. 
In älteren Samen, die nach dem Verhalten normaler OenotheraSamen schon vielzellige Embryonen und reiches, schon durch Zellwände

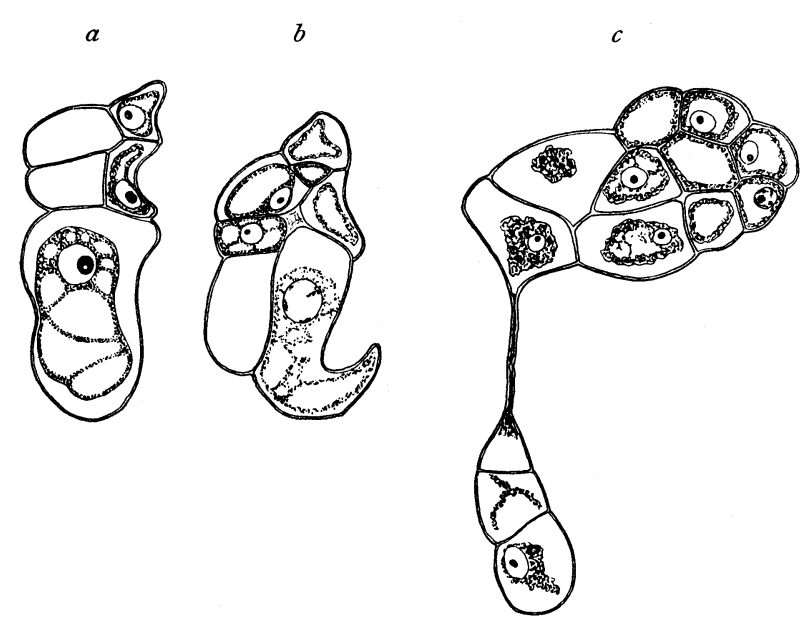

iig. 9. 0. muricata $\times$ biennis. Kranke Embryonen aus albreifen Samen. $a$ und $b$ aufeinanderfolgende Längsschnitte us demselben Embryo. A pochr. $2 \mathrm{~mm}$, Ok. 6; auf $1 / 2$ verkl.

gefächertes Endosperm enthalten sollten, ist die Samenschale wohl ziemlich normal entwickelt (Textfigur 8), aber die Embryosackwand ist meistens verschwunden, und vom Endosperm sind nur noch krümelige Plasmareste vorhanden. Die innersten Schichten des Nucellus sind zertört, die Zellen zerdrückt und plasmaleer, ihre Wände mehr oder weniger erquollen, so daß der ursprünglich vom Embryosack eingenommene

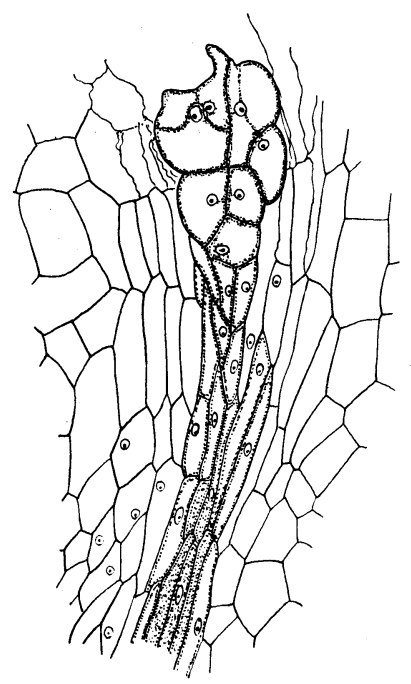

Fig. 10.

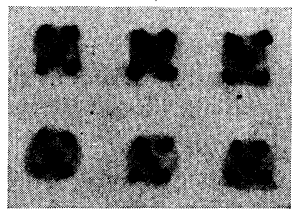

Fig. 11.

Fig. 10. 0. muricata. Zellen des Nuzellus am Chalazapol des Embryosackes, mehrere Wochen nach der Bestäubung mit dem Pollen von O. biennis. Apochr. $2 \mathrm{~mm}$, Ok. 2; auf $1 / 2$ verkl.

Fig. 11. Oben Samen von 0. muricata $x$ muricata, unten Samen von 0 . muricata $x$ biennis. Natürl. Größe.

iaum oft sehr verkleinert ist. Der Embryo ist zu einem unregelmäßig eformten, wenigzelligen Körper herangewachsen (Textfig. 9 ; im Maximum 
wurden etwa 15-20 Zellen gezählt). Die Zellen sind groß, verhältnismäßig dickwandig, plasmaarm, oft von außen eingedellt oder ganz zusammengedrückt und abgestorben. In noch älteren Samen ist auch vom Embryo nichts mehr zu finden. Die Nucelluszellen, die an den Chalazapol des Embryosackes angrenzen, wachsen mitunter beträchtlich heran (Textfig. 10), was in gesunden Samen nie beobachtet wurde. Bei der Reife schrumpfen die Samen der Kreuzung und sind dann von gesunden, nach spontaner Bestäubung gebildeten Samen der O. muricata-Venedig leicht zu unterscheiden (Textfig. 11). Doch geht die Entwicklung der Samenschale bei den kranken Samen so weit, daß das Stehenbleiben des Embryo und des Endosperms nicht als ein Verhungern aufgefaßt werden kann.

Die Auflösung der inneren Schichten des Nucellus ist keine Besonderheit, die den kranken, tauben Samen eigentümlich ist, sie findet sich auch in gesunden Samen in der Umgebung des heranwachsenden, von Endosperm erfüllten Embryosackes (vgl. unten bei O. Lamarckiana $X$ biennis). In gesunden Samen möchte man an eine verdauende Wirkung des sich ausdehnenden Endosperms denken. Ob in den kranken Samen das Nucellusgewebe sich selbst verdaut oder doch noch vom absterbenden Embryosack Enzyme ausgeschieden werden, läßt sich nicht entscheiden. Es liegen hier ähnliche Erscheinungen vor wie bei dem ohne Befruchtung sich entwickelnden Endosperm von Ficus carica, das trotz dem Fehlen eines Embryo aufgelöst wird, also sich selbst verdaut (Tischler 1913, pag. 27, 29).

Daß reziproke Kreuzungen, an denen 0 . biennis oder muricata oder beide beteiligt sind, verschiedenes Resultat haben, ist durch de Vries genügend bekannt. Auch bei den Rassen, die de Vries verwendet hat, sind die Bastarde 0 . biennis $\times$ muricata und 0 . muricata $\times$ biennis einander sehr unähnlich. Dieser Unterschied geht bei 0 . muricataVenedig noch weiter: wenn diese Art Mutter ist und O. biennis Vater, so ist das Produkt der Kreuzung gar nicht entwicklungsfähig, während das der reziproken Kreuzung wenigstens eine Zeitlang normal sich zu entwickeln scheint. $\mathrm{Ob} O$. biennis mit muricata-Venedig bestäubt keimfähige Samen liefert, soll noch geprüft werden.

\section{f) O. muricata-Venedig $+9 \times$ Lamarckiana 0 .}

Es sind nur sehr junge Samen untersucht worden, die wie bei der Kreuzung mit O. biennis alle kranke Embryosäcke enthielten. Die Embryonen scheinen das Wachstum noch früher einzustellen als bei der 
Kreuzung muricata $\times$ biennis. - Die Samen der reziproken Kreuzung sind verloren gegangen.

g) O. biennis 우 $\times$ Lamarckiana $\delta$.

Die Befruchtungsgeschichte stimmt in allen Punkten mit der für O. biennis $\times$ muricata geschilderten überein. Ein befruchtetes, schon herangewachsenes Ei mit zweinukleoligem Kern und einen zweizelligen Embryo gibt die Textfig. 12.

Die erste Teilung der befruchteten Eizelle ist nicht beobachtet worden, wohl aber mehrfach die zweite; vgl. die deutliche Äquatorial-

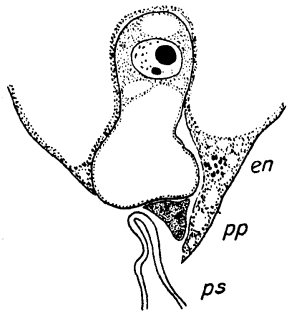

Fig. 12. 0. biennis $\times$ Lamarckiana. Befruchtetes $\mathrm{Ei}$ und zweizelliger Embryo. A pochr. $2 \mathrm{~mm}, 0 \mathrm{k}$. 6 ; auf $2 / 3$ verkl. $\not p$ (der dreieckige Körper) Pollenschlauchplasma in der Synergide.

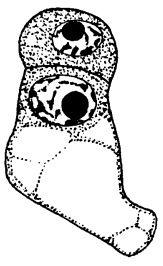

Be-

-
platte Taf. XII, Fig. 20 mit 15 (oder 14?) Chromosomen und die Prophase im Suspensor mit sehr großen Chromosomen (Fig. 17). In etwas älteren Embryonen wurden alle Stadien der Kernteilung gefunden (Fig. 18, 19, 22, 23). Die Chromosomenzahl ist immer ungefähr 14; die sichere Entscheidung zwischen 13,14 und 15 ist meistens kaum möglich.

Auch im Endosperm sind ungefähr 14 Chromosomen zu zählen (Fig. 21).

Kranke Embryonen sind nicht beobachtet worden.

h) O. Lamarckiana $9 \times$ biènnis $\delta$.

Die Embryo- und Endospermbildung ist oft normal, und die Chromosomenzahl ist etwa 14 (Fig. 24). Ebenso oft entwickeln sich aber Embryo und Endosperm ähnlich unvollständig wie bei 0 . muricata-Venedig $\times$ biennis. Die Textfig. 13 gibt einen gesunden Embryo $a$ und drei kranke, von denen $b$ ungefähr so groß ist wie der gesunde; die Embryonen stammen aus Fruchtknoten, die 14 Tage nach der Bestäubung fixiert wurden. Die Zellen der kranken Embryonen sind groß, plasmaarm, besitzen einen großen Kern und teilen sich sehr langsam; Mitosen sind überhaupt nicht angetroffen worden. Noch auffälliger ist wieder die Störung der Endospermbildung. In dem Embryosack, aus dem der vierzellige Embryo Fig. 13d stammt, waren nur zwei Endospermkerne zu finden, von denen der eine, auf dem Embryo sitzende gezeichnet ist. Neben dem Embryo Fig. $13 b$ fanden sich im Präparat sechs Endosperm- 
kerne; der Embryosack war aber nicht ganz vollständig, und vielleicht waren in Wirklichkeit mehr Kerne vorhanden. Mehr als acht Kerne dürften aber kaum irgendwo vorkommen. Diese Endospermkerne sind noch größer als bei 0 . muricata $\times$ biennis, gewöhnlich sind sie riesenhaft und enthalten mehrere vakuolige Nukleolen. Der Endospermkern in Fig. $13 e$ ist so dick wie der dreizellige Kopf des zugehörigen Embryo (außerdem war nur noch ein zweiter Kern vorhanden), der Kern Fig. $13 c$ ist fast so lang wie der zugehörige Embryo Fig. 13c. 3 Wochen nach der Bestäubung sind die gesunden Embryonen teilweise schon zweilappig, die gesunden Endosperme sind größtenteils durch Wände gefächert
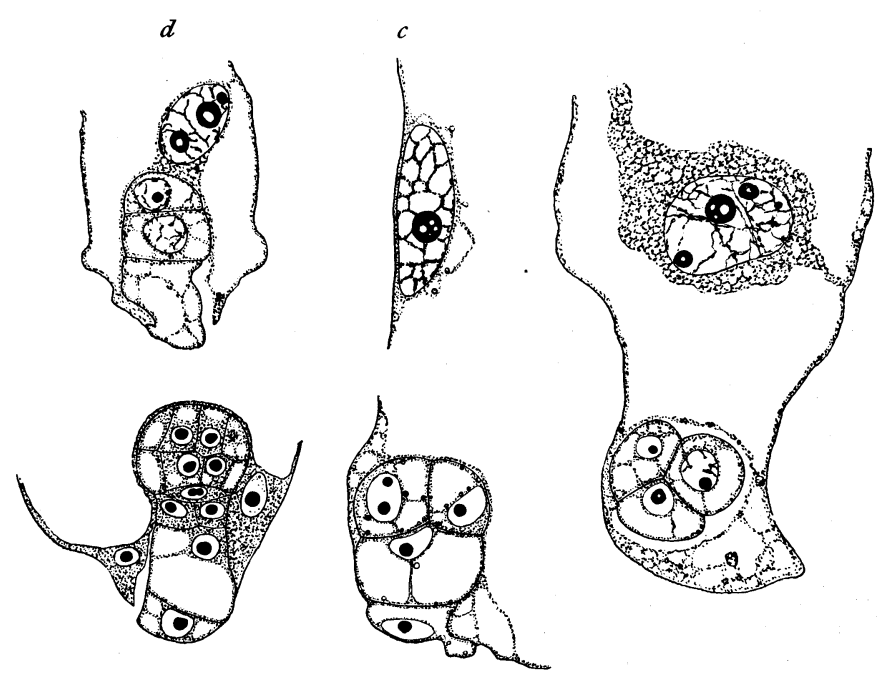

$a$

$b$

Fig. 13. O. Lamarckiana $\times$ biennis. $a$ gesunder Embryo. $b, d, e$ kranke Embryonen, $d$ und $e$ mit je einem kranken Endospermkern. $c$ kranker Endospermkern, zu $b$ gehörig. Apochr. $2 \mathrm{~mm}$, Ok. 6; auf $1 / 2$ verkl.

und füllen die Embryosäcke aus. Die kranken Embryonen sind gar nicht mehr gewachsen und mehr oder weniger verschrumpft. Der Beginn der Auflösung des Nucellusgewebes, wie oben von 0 . muricata $\times$ biennis beschrieben, ist in gesunden und kranken Samen zu sehen.

Die Chromosomenzahl konnte in den kranken Embryonen und Endospermen nicht festgestellt werden.

Gesunde und kranke Embryosäcke scheinen in gleicher Zahl vorzukommen. In einer Schnittserie desselben Materials finden sich nämlich 21 gesunde und 19 kranke Embryosäcke. Wenn in der fünften Serie von verhältnismäßig weit entwickelten Samen neben 10 gesunden 
nur ein kranker zu finden ist, so liegt das vielleicht daran, daß unwillkürlich zunächst die am besten entwickelten Samen im Paraffin zusammengelegt wurden. Denn die kranken Samen fangen bald an im Wachstum hinter den gesunden zurückzubleiben.

Zur sicheren Ermittlung des Zahlenverhältnisses wurden die aus einer Anzahl reifer Kapseln ausgefallenen samen aufgeweicht und mit Nadeln geöffnet. Es waren 46 gesunde Samen mit großem Embryo und 50 taube Samen, in denen bei schwacher Vergrößerung kein Embryo zu entdecken war. Dabei ergab sich, daß in der überwiegenden Mehrzahl die gesunden Samen bedeutend größer waren als die tauben. Für die

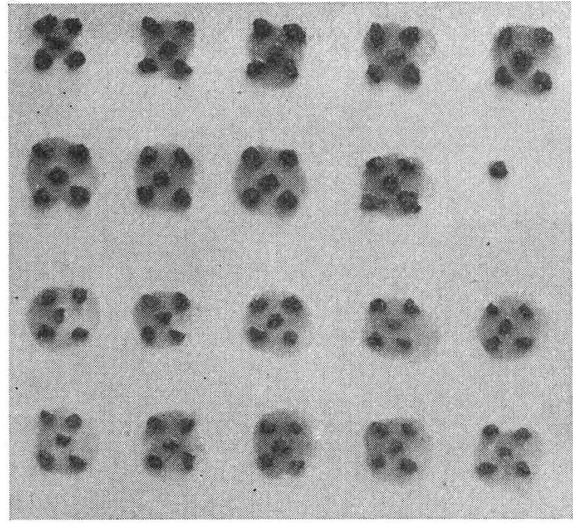

Fig. 14. 0. Lamarckiana $\times$ biennis. Reife Samen, oben 46 gesunde, unten 50 taube. Natürl. Größe.

Auszählung des übrigen Samenmaterials (gerade hier waren leider ganz wenige Kapseln geerntet worden) war deshalb die Öffnung nicht nötig. Tatsächlich ließen sich die trockenen Samen zum allergrößten Teil mit Sicherheit in zwei Gruppen sondern, in große, pralle, und in kleinere, verschrumpfte, flache; in zweifelhaften Fällen wurden die Samen aufgeweicht und geöffnet. Das Ergebnis der Zählung an der ersten Probe gibt die Fig. 14 (nach trockenen Samen aus einer anderen Frucht).

Von den in vier (schon teilweise entleerten) Kapseln enthaltenen Samen waren gesund: $36 \quad 22 \quad 44 \quad 25$, krank: $31 \quad 27 \quad 36 \quad 25$.

Alles in allem waren von 342 Samen 173 gesund und 169 taub. Gute und taube Samen wechseln in den Kapselfächern ziemlich regelmäßig ab. Die Einfachheit des Zahlenverhältnisses $(1: 1)$ und die Übereinstimmung zwischen den verschiedenen Früchten macht es so gut wie sicher, daß wir es mit einer genotypisch bedingten Erscheinung zu tun haben.

\section{Embryologische Beiträge zur Vererbungslehre.}

a) O. (Lamarckiana $\times$ biennis) ein Zwillingsbastard.

Die Kreuzung 0 . biennis $ᄋ \times$ Lamarckiana $O^{\top}$ gibt nach der Entdeckung von de Vries in $F_{1}$ zwei verschiedene Formen, „Zwillinge“, 
die gewöhnlich in ungefähr gleicher Zahl auftreten (de Vries 1913, pag. 121ff.) und die de Vries als Laeta und Velutina bezeichnet hat. Die reziproke Kreuzung 0 . Lamarckiana $q \times$ biennis $0^{x}$, mit der wir uns gegenwärtig beschäftigen, liefert nach den Versuchen von de Vries und anderen immer eine einförmige Nachkommenschaft. Aber wie-wir jetzt aus dem embryologischen Befund wissen, verhält sich diese Kreuzung im Grunde nicht anders als die umgekehrte, bei der O. Lamarckiana Vater ist. Es entstehen wieder in gleichen Zahlen zwei $\mathrm{F}_{1}$-Formen, eine entwicklungsfähige, der lang bekannte, ,einförmige“ Bastard, und eine entwicklungsunfähige Form, die auf dem Stadium sehr junger Embryonen abstirbt und die wir deshalb als Keimpflanze nie zu sehen bekommen. Die Einförmigkeit der Kreuzung O. Lamarckiana $\times$ biennis wird vorgetäuscht durch die Ausschaltung des einen von zwei verschiedenen Zygotentypen. Auch O. Lamarckiana $X$ biennis ist ein Zwilling, aber einer, der seinen Bruder sehr früh verliert.

Eine biennis-Form, die de Vries als biennis-Chicago bezeichnet, gibt mit O. Lamarckiana tatsächlich bei Kreuzung in beiden Richtungen Zwillingsbastarde in der ersten Generation. Gerade die Kreuzung, die bei unserer biennis scheinbar einförmig ist, nämlich Lamarckiana $\times$ biennis, spaltet bei biennis-Chicago in die Typen laeta und velutina (de Vries 1913, pag. 124). Die reziproke Verbindung dagegen liefert ein anderes Zwillingspaar, densa und laxa (ebenda pag. 144). Mit der densa-laxa-Spaltung haben wir das Ergebnis der Kreuzung zwischen der Lamarckiana o und unserer biennis o wohl am ehesten zu vergleichen. Die entwicklungsfähige Form sieht nach de Vries und anderen Autoren dem Vater, der biennis, sehr ähnlich und besitzt viel rotes Pigment, sie entpricht also dem Zwilling laxa, und die ausgeschaltete Kombination ist als Zwilling densa aufzufassen. Die densa aus biennis-Chicago ist nach de Vries (pag. 149) schwach und dünnstengelig, die laxa ist kräftig und hochwüchsig. Bei unserer biennis-Form ist die densa noch weiter geschwächt und überhaupt nicht lebensfähig. Vergleichen wir den ,verwaisten“ laxa-Zwilling aus Lamarckiana $\times$ biennis mit den Zwillingen aus biennis $\times$ Lamarckiana, so entspricht er nach der biennisÄhnlichkeit und nach der Pigmentierung der velutina, nicht der laeta. Auch de Vries vergleicht laxa mit velutina und densa mit laeta (pag. 149).

Die aus O. Lamarckiana öfters hervorgehende Mutante O. lata wird von unserer biennis o ${ }^{*}$ in zwei in gleichen Zahlen auftretende Bastardtypen gespalten, in eine lata-Form, die gleichzeitig Merkmale von biennis hat, und in eine Form, die von dem Bastard Lamarckiana $\times$ Flora, Bd. 107. 
biennis nicht zu unterscheiden ist (de Vries 1913, pag. 251). Der bei der Kreuzung Lamarckiana $\times$ biennis ausfallende Zwilling entspricht also dem lata-Zwilling aus der Kreuzung lata $\times$ biennis.

Mehr als die zwei Formen laeta und velutina gehen aus der Kreuzung O. biennis $\times$ Lamarckiana in $\mathrm{F}_{1}$ kaum hervor. Denn kranke Embryosäcke in den Präparaten und taube Samen (bei einer Untersuchung von 263 reifen Samen) wurden hier nicht beobachtet, es scheint also keine Kombinationen zu geben, die als junge Zygoten ausgeschaltet werden. Wenn die Typen laeta und velutina sich in mehrere Formen auflösen lassen, wie L o ts y meint (1912, pag. 327), so hat das mit unserer Frage nichts $\mathrm{zu} \operatorname{tun}^{1}$ ).

Nach de Vries (1913, z. B. pag. 118, 119, 124, 125) und nach Honing („Doppelnatur", pag. 235) findet sich die laeta oft in beträchtlich kleinerer Zahl als die velutina oder umgekehrt. Ebenso fand de Vries „oft viel mehr Exemplare von der laxa als von der densa" (1913, pag. 145). Das kann aber mit verschiedenem Keimungsvermögen der beiden zusammengehörigen Bastardrassen zusammenhängen. $\mathrm{Ob}$ es wirklich Linien der O. Lamarckiana gibt, die die Zwillingsbastarde regelmäßig in ungleicher Zahl erzeugen, wird man jetzt am einfachsten an der Kreuzung Lamarckiana $\times$ biennis prüfen, weil man hier nicht auf die gekeimten Samen angewiesen ist, sondern die Samen als solche, unbekümmert um ihre Keimfähigkeit, auszählen kann.

De Vries hat, um den verschiedenen Erfolg der beiden reziproken Kreuzungen zwischen 0 . Lamarckiana einerseits und 0 . biennis oder muricata andererseits zu erklären, annehmen müssen, die Lamarckiana sei wohl immer (passiv) ,,spaltbar", aber die Spaltung in zwei $F_{1}$-Formen werde nur dann ausgeführt, wenn die zur Kreuzung verwendete Art das entsprechende (aktive) ,Spaltungsvermögen“ besitzt (1913, pag. 133, 157). Durch die ,isogamen“ Arten (O. Cockerelli, Hookeri, strigosa) wird die Lamarckiana immer in laeta und velutina gespalten, einerlei ob von der betreffenden Art der Pollen oder die Eizellen zur Verbindung mit Lamarckiana verwendet werden. Unter den ,heterogamen“ Spezies verhält sich gleich nur O. biennis-Chicago, die als Vater mit den Eizellen von Lamarckiana die .üblichen Formen laeta und velutina gibt, als Mutter ein anderes Zwillingspaar, densa und laxa. Die übrigen hetero-

1) Lotsy spricht übrigens von $F_{2}$. Wenn das nicht ein Druckfehler für $F_{1}$ ist, so ist nicht recht zu verstehen, was er im Auge hat. Denn von der $F_{2}$ von 0 . biennis $\times$ Lamarckiana hat de Vries doch nie angegeben, daß sie ,in 0 . laeta und 0 . velutina im Verhältnis 1:1 spaltet", vielmehr sollen beide Zwillinge bei Selbstbestäubung konstant sein. 
gamen Arten, z. B. biennis und muricata, sollen nur in ihren Eizellen das Vermögen besitzen die Lamarckiana zu spalten, im Pollen nicht. Für biennis ist nun aber nachgewiesen, daß dieser Unterschied zwischen Pollen und Eizellen nicht besteht, und O. muricata wird sich wohl ebenso verhalten. Wir dürfen deshalb wohl annehmen, daß O. Lamarckiana nicht bloßimmer potentia spaltbar ist, sondern bei Bastardierung in $\mathrm{F}_{1}$ auch immer wirklich gespalten wird.

b) Zur Frage der Heterozygotennatur der O. Lamarckiana.

Wenn wir das jetzt ziemlich gleichförmig erscheinende Verhalten der O. Lamarckiana bei der Kreuzung mit O. biennis und anderen Arten Spaltung der $\mathrm{F}_{1}$-Generation in zwei gleich häufig auftretende Bastardtypen - auf das Schema einer Mendel-Spaltung bringen wollen, müssen wir annehmen, die 0 . Lamarckiana sei in einem hier maßgebenden Faktor heterozygotisch. Die zweierlei Keimzellen, die sie bildet, geben beim Zusammentreffen mit den Keimzellen von biennis entweder laeta bzw. densa oder velutina bzw. laxa. Bei Selbstbestäubung der 0 . Lamarckiana müßten außer 50\% Heterozygoten zweierlei homozygotische Typen in je $25 \%$ auftreten. Falls diese Homozygoten zur Entwicklung kämen, müßten sie bei Kreuzung mit biennis einförmige $F_{1}$ geben. Aus der Homozygote mit velutina-Eigenschaft ginge nur velutina hervor, wenn biennis Mutter ist; und der bekannte, jetzt als laxa zu bezeichnende Bastard, wenn biennis Vater ist. Die Homozygote mit laeta-Eigenschaft würde als Vater nur laeta-Bastarde erzeugen, und als Mutter würde sie nur taube Bastardsamen bringen. Bis jetzt ist aber nie davon berichtet worden, daß die Kreuzung biennis $\times$ Lamarckiana in $\mathrm{F}_{1}$ einförmig sein kann, und ebensowenig, daß die Kreuzung Lamarckiana $\times$ biennis nicht gelingt. Lamarckiana hat sich also immer als heterozygotisch erwiesen, und ebenso die meisten ihrer Mutanten, z. B. nanella und rubrinervis, die ebenfalls bei Kreuzung mit biennis usw. in laeta und velutina spalten. Dann müssen aber - angenommen immer, daß die in Rede stehenden Erscheinungen sich den Me ndelschen Regeln fügen - die Homozygoten von beiderlei Art regelmäßig ausgeschaltet werden. Wenn das auf frühem Stadium geschieht, ähnlich wie bei dem densa-Zwilling aus $O$. biennis, muß es sich leicht ermitteln lassen.

Spontan bestäubte Fruchtknoten von Lamarckiana aus derselben Population, die zu den Kreuzungen gedient hatte, und in deren Nachbarschaft weit und breit keine anderen Önotheren standen, wurden geschnitten und auf kranke Embryosäcke untersucht. In jungen Stadien 
waren gewisse Embryonen wieder weit hinter der Mehrzahl zurück, und das Endosperm in den betreffenden Embryosäcken zeigte dieselben Störungen, wie sie oben mehrfach beschrieben worden sind. Die gesund erscheinenden Emrbyosäcke waren bedeutend zahlreicher als die kranken. Von fast reifen Samen desselben Materials, die wie die jungen Stadien fixiert worden waren, wurde zunächst eine Probe mit der Hand geschnitten, im ganzen 66 Stück. Davon enthielten 25 vollentwickelte Embryonen; in 21 Samen waren die Embryonen noch sehr klein und kugelig, das Endosperm überzog als dünner Belag die Embryosackwand; in 20 Samen waren entweder gar keine oder winzig kleine, wenigzellige Embryonen zu finden und auch vom Endosperm höchstens Spuren.

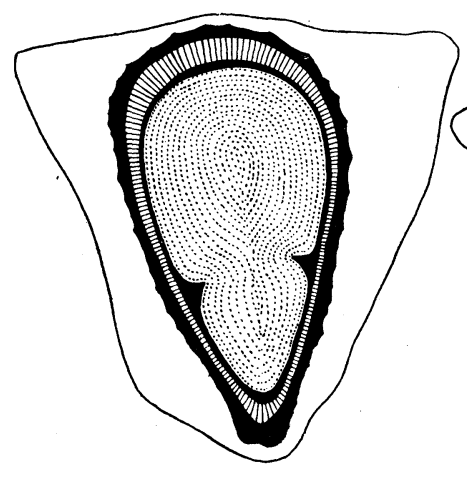

$a$

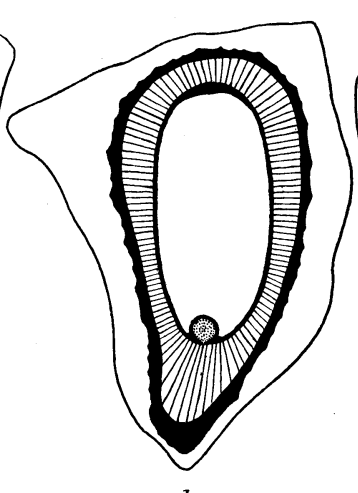

$b$

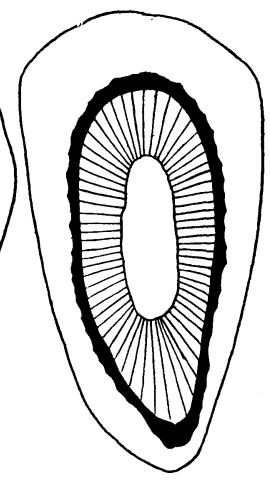

c

Fig. 15. O. Lamarckiana $\times$ Lamarckiana. Drei ziemlich reife Samen im Längsschnitt. $a$ mit gesundem Embryo und gesundem Endosperm. $b$ mit sehr kleinem kugeligem Embryo und dünner Endospermschicht. $c$ ohne Embryo und ohne Endosperm. Embryo punktiert, Endosperm schwarz. Nuzellus schraffiert, Hartschicht der Samenschale schwarz.

An Mikrotomschnitten wurden diese drei Kategorien von Samen genauer untersucht (vgl. Textfig. 15). Die Samenschale ist überall ziemlich gleich entwickelt, nur die zweischichtige Hartzone an der Innenseite der Testa ist bei den ganz tauben Samen mitunter dünnwandiger als bei den übrigen. Vom Embryo und vom Endosperm sind in den ganz tauben Samen nur noch Spuren zu finden, das Nucellusgewebe ist immer angegriffen, mitunter zu einem großen Teil aufgelöst. In den Samen, die kleine kugelige Embryonen enthalten, ist eine großenteils einfache Schicht von Endospermzellen vorhanden, das Nucellusgewebe ist erhalten. Die vollkommen ausgebildeten Embryonen liegen in einer sehr dünnen, großenteils einfachen Schicht von Endosperm, sie haben also das Endosperm, das in früheren Stadien den Embryosack- 
raum ausfüllte, zum größten Teil aufgezehrt; auch vom Nucellus ist nur noch eine wenige Zellen dicke Schicht erhalten.

O. Lamarckiana erzeugt also bei Selbstbestäubung tatsächlich kranke Samen. Die nicht normalen Embryonen und Endosperme bleiben auf mindestens zwei deutlich unterscheidbaren Stufen stehen. Um ein Verhungern der nicht normalen Embryosäcke kann es sich auch hier kaum handeln, weil die Testa sich vollkommen ausbildet. Die nicht zur Reife gelangenden Embryonen und Endosperme stellen also wohl Biotypen dar, die nicht entwicklungsfähig sind. Die tauben Samen beiderlei Art sind miteinander etwa doppelt so zahlreich als die gesunden. Wenn eine Mendel-Spaltung vorliegt, bei der die Heterozygoten sich normal entwickeln, die Homozygoten (wenigstens zum Teil) als taube Samen auftreten, dann ist demnach die untersuchte Linie von O. Lamarckiana mindestens doppelt heterozygotisch. Wäre sie monohybrid, so müßten gesunde und taube Samen ja in gleicher Zahl gebildet werden.

Zygotische Kombinationen, die nicht eigens für embryologische Untersuchung fixiert worden, waren nur als reife trockene Samen zu erhalten. Wenn solche untersucht werden sollten, war es zunächst nötig zu wissen, ob Kombinationen, die nach dem mikroskopischen Befund nur als gesunde Embryonen erscheinen, auch in den reifen Kapseln nur als gut ausgebildete Samen auftreten. Tatsächlich waren die Samen, die an O. biennis im Freien bei spontaner Bestäubung gebildet wurden, zum allergrößten Teil gut entwickelt und mit fertigem Embryo versehen. Doch fehlen sehr kleine, verschrumpfte Samen, noch kleiner als die tauben Samen von 0. Lamarckiana $\times$ biennis, nicht vollständig. Sie sind von dem feinen Pulver der sterilen Samenanlagen, die man in allen Oenothera-Früchten findet, fast immer sicher zu unterscheiden, bedeutend größer, auch dunkler gefärbt, aber doch ganz flach und leicht. Vielleicht sind es befruchtungsfähige, aber unbefruchtet gebliebene Samenanlagen, die trotz dem Ausbleiben der Befruchtung etwas heranwachsen. Jedenfalls dürfen solche ganz kleinen Samen nicht zu den tauben mit früh absterbendem Embryo gezählt werden; allerdings ist es mitunter schwer, zwischen den beiden Kategorien eine sichere Grenze zu finden. Bei der Probe von O. biennis kamen auf 204 gute Samen 7 ganz kleine; große taube Samen fehlten. Bei spontan bestäubter O. muricata-Venedig kamen auf 308 gute Samen 27 sehr kleine. Bei der Kreuzung O. biennis $\times$ Lamarckiana wurden 263 gesunde Samen gezählt (die laeta und velutina geben müssen) und $10 \mathrm{ganz}$ kleine, blasse. Das Ergebnis entsprach also, von den noch etwas zweifelhaften 
ganz kleinen Samen abgesehen, der Erwartung, und demnach scheint es erlaubt, für Samen, die im reifen Zustand bei beträchtlicher Größe taub sind, Störung der Embryoentwicklung nach den oben geschilderten Mustern anzunehmen. Treten die tauben Samen in festen Zahlenverhältnissen auf, so läßt sich kaum etwas dagegen einwenden, daß man sie als die Repräsentanten bestimmter genotypischer Kombinationen ansieht.

Um verschiedenerlei Material vergleichen zu können, habe ich einige Vererbungsforscher, die Önotheren züchten, um Samen von 0. Lamarckiana und von gewissen Bastardrassen gebeten. Den Herren Prof. de V́ries, Dr. R. R. Gates und Dr. Heribert-Nilsson, die in der Lage waren meine Bitte zu erfüllen und mir in der liebenswürdigsten Weise reichliches Material zur Verfügung stellten, möchte ich auch hier meinen verbindlichsten Dank sagen. Herr Prof. de Vries teilte mir überdies mit, daß O. Lamarckiana nach seinen Erfahrungen gewöhnlich nur zu einem Drittel keimfähige und zu zwei Dritteln leere Samen habe und daß O. rubrinervis und nanella fast genau zur Hälfte keimen, während O. gigas und die wildwachsenden Arten fast zu 100\% keimfähig seien.

Herr Heribert-Nilsson machte mich auf einen kurzen Bericht (1913) über seine neueren Züchtungsergebnisse aufmerksam, die für unsere Frage von der größten Wichtigkeit sind. Daß die weißnervigen Individuen von $O$. Lamarckiana, die sich von den rotnervigen auch im Habitus unterscheiden, in der Nervenfarbe bei Selbstbefruchtung konstant, also Homozygoten sind, hat Heribert-Nilsson schon früher (1912, pag. 100) mitgeteilt. Seitdem hat sich weiter herausgestellt, daß die rotnervigen Individuen bei Selbstbefruchtung immer Weißnerven abspalten; von 23 roten Linien haben alle Spaltung gezeigt. Die Rotnerven sind demnach immer Heterozygoten in bezug auf die Nervenfarbe und tragen das dominante Merkmal zur Schau. Von den Homozygoten sind nur die mit dem rezessiven Merkmal, die Weißnerven, lebẹnsfähig; die mit dem Faktor für Rotnervigkeit doppelt ausgestatteten Individuen kommen nicht zur Entwicklung, sie werden auf irgend einem Stadium vor der Keimung eliminiert. Tatsächlich erfolgt die Spaltung in den meisten rotnervigen Linien nach 2:1, wodurch die Frage mit aller Bestimmtheit entschieden ist. Solche Fälle sind ja nicht mehr neu.

Was die Rotnerven betrifft, ist also ihre Heterozygotennatur schon bewiesen. Aber auch die in bezug auf die Nervenfarbe homozygotischen Weißnerven müssen in dem Faktor, der die Spaltung in laeta und velutina bedingt, heterozygotisch sein. Ho ning (,Doppelnatur", pag. 261) gibt nämlich an, daß er aus 2jähriger $O$. Lamarckiana in $F_{1}$ Zwillinge 
bekommen hat, und seine 2jährigen Individuen waren alle weißnervig. Die Zwillingsspaltung ist also von dem Faktor für Nervenfarbe unabhängig.

Nehmen wir den einfachsten Fall an, wie er nach den von de Vries und von Heribert-Nilsson beobachteten Spaltungszahlen ganz wohl vorliegen kann: Die Spaltung in laeta und velutina wird durch einen einzigen Faktor bedingt, ebenso wie die Nervenfarbe von einem einzigen Faktor abhängt. Dann ist die weißnervige O. Lamarckiana, weil sie wenigstens den Faktor für Nervenfarbe in homozygotischem Zustand besitzt, in den uns interessierenden Punkten monohybrid, die rotnervige ist dihybrid. Der laeta-velutina-Faktor ist immer in heterozygotischem Zustand vorhanden, als Ll; das L mag Symbol für laeta sein. Eine biennis $\times$ Lamarckiana-Zygote, die den Faktor L besitzt, wird laeta, eine andere, die aus dem Pollen von Lamarckiana den Faktor l erhält, wird velutina.

Der Faktor für Rotnervigkeit sei R, für Weißnervigkeit $r$, dann sind die Formeln der beiden durch die Nervenfarbe unterschiedenen Formen: Rotnervig $\mathrm{Rr} \mathrm{Ll}$, weißnervig $\mathrm{rr} \mathrm{Ll}$. Lebensunfähig sind alle Zygoten mit den homozygotischen Faktoren RR, LL, 11.

Die Weißnerven geben bei Selbstbefruchtung:

$$
1 \text { rr LL + } 2 \text { rr Ll }+1 \text { rr ll, }
$$

sie müssen also zur Hälfte gesunde und zur Hälfte taube Samen bringen.

Die dihybriden Rotnerven spalten auf 16 Individuen in

4 Rr Ll
2 rr Ll
2 Rr LL
2 Rr ll
2 RR Ll
1 RR LL
1 RR ll
1 rr LL
1 rr ll.

Lebensfähig sind nur die beiden ersten Gruppen. In diesen sind die Rotnerven doppelt so zahlreich wie die Weißnerven, wie HeribertNilsson es gefunden hat. Die gesunden Samen miteinander müssen zu den tauben im Verhältnis von $6: 10$ stehen, und das ist ungefähr das Verhältnis $1: 2$, das de Vries im allgemeinen beobachtet hat (vgl. oben). 
Die Auszählung der gesunden und der tauben Samen war bei den meisten Materialien viel schwieriger als bei der Kreuzung O. Lamarckiana $\times$ biennis. Große Samen sind oft taub, sehr kleine Samen können einen fertig entwickelten Embryo einschließen; das ist ein weiterer Beweis dafür, daß die tauben Samen kranke und nicht etwa ungenügend ernährte Embryonen enthalten. Für O. Lamarckiana z. B. kann aus diesem Grund die Spaltung nicht makroskopisch in der Weise vorgezeigt werden, wie es für die Kreuzung 0 . Lamarckiana $\times$ biennis möglich war (vgl. pag. 130). Um nicht alle keimfähigen Samen der Untersuchung zu opfern, wurden entweder die Samen zunächst zur Keimung ausgelegt und erst die. nichtgekeimten geöffnet, oder es wurden nur Proben des Materials Stück für Stück geöffnet, oder es wurden bei leichter zu beurteilenden Materialien nur die verdächtigen Samen geöffnet. Das Ergebnis der Zählungen gibt die folgende Tabelle. Ein Fragezeichen hinter der Zahl der gesunden Samen bedeutet, daß nicht alle geöffnet wurden, daß also noch taube Samen darunter sein können. Die ganz kleinen Samen (vgl. pag. 135) wurden so gut es ging ausgeschieden und sind in der Tabelle nicht aufgeführt.

Tabelle 1.

\begin{tabular}{|c|c|c|c|c|c|c|}
\hline Material & Herkunft & $\begin{array}{l}\text { Gute } \\
\text { Samen }\end{array}$ & $\begin{array}{l}\text { Taube } \\
\text { Samen }\end{array}$ & $\begin{array}{c}\text { Theoretische } \\
\text { Zahlen }\end{array}$ & $\begin{array}{l}\text { Mitt- } \\
\text { lerer } \\
\text { Fehler }\end{array}$ & $\begin{array}{c}\text { Spal- } \\
\text { tungs- } \\
\text { ver- } \\
\text { hältnis }\end{array}$ \\
\hline 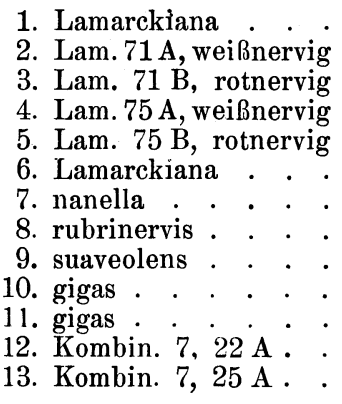 & 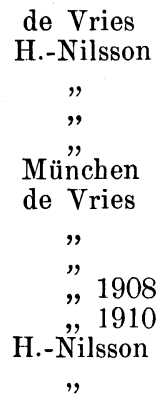 & $\begin{array}{r}109 \\
62 \\
34 \\
22 \\
30 \\
25 \\
117 \\
140 \\
97 \\
47 \\
88 \\
77 ? \\
154 ?\end{array}$ & $\begin{array}{r}151 \\
62 \\
68 \\
75 \\
65 \\
41 \\
133 \\
130 \\
103 \\
7 \\
33 \\
0 \\
2\end{array}$ & $\begin{aligned} 97,5 & : 162,5 \\
62 & : 62 \\
38,25 & : 63,75 \\
24,25 & : 72,75 \\
35,625 & : 59,375 \\
24,75 & : 41,25 \\
125 & : 125 \\
135 & : 135 \\
100 & : 100\end{aligned}$ & $\begin{array}{l} \pm 7,8 \\
\pm 4,89 \\
\pm 4,27 \\
\pm 4,72 \\
\pm 7,91 \\
\pm 8,16 \\
\pm 7,07\end{array}$ & $\begin{array}{l}6: 10 \\
2: 2 \\
6: 10 \\
1: 3 \\
6: 10 \\
6: 10 \\
2: 2 \\
2: 2 \\
2: 2\end{array}$ \\
\hline
\end{tabular}

Das nach dem oben gesagten für weißnervige O. Lamarckiana zu erwartende Verhältnis $1: 1$ findet sich tatsächlich bei Nr. 3 der Linie $71 \mathrm{~A}$ von Heribert-Nilsson. Auch die beiden rotnervigen Linien $71 \mathrm{~B}$ und $75 \mathrm{~B}$ zeigen ungefähr das erwartete Spaltungsverhältnis $6: 10$. Die weißnervige Linie $75 \mathrm{~A}$ dagegen, eine Schwesterlinie von $75 \mathrm{~B}$ (so wie $71 \mathrm{~A}$ und $\mathrm{B}$ Schwesterlinien, d. h. aus einem selbstbefruchteten rot- 
nervigen Exemplar hervorgegangen sind), hatte nur etwa ein Viertel gute Samen. Hier muß also eine Komplikation vorliegen, die in der zugehörigen rotnervigen Linie sich nicht manifestiert hat; verständlicher wäre es, wenn die rotnervige Schwesterlinie noch weniger als $25 \%$ gute Samen hätte. Die beiden anderen Materialien von Lamarckiana, deren Nervenfarbe nicht bekannt ist (Nr. 1 und 6), spalten etwa im Verhältnis $6: 10$. - Die Abweichung der gefundenen von den theoretisch erwarteten Zahlen ist bei Nr. 1 und 5 beträchtlich. Ob das der Ausdruck einer komplizierteren Heterozygotie ist, müssen weitere Studien ergeben.

Nach der obenerwähnten brieflichen Mitteilung von Herrn Prof. de Vries dürfte das Spaltungsverhältnis $6: 10$, das zu 37,5\% gesunder Samen führt, bei O. Lamarckiana am häufigsten vorkommen. In der Literatur sind nur Angaben über Keimprozente zu finden, die natürlich in der uns interessierenden Frage wichtige Fingerzeige geben, aber die Feststellung des Zahlenverhältnisses zwischen vollentwickelten und tauben Samen nicht ersetzen können, weil die gesund erscheinenden Samen oft nicht alle keimen. Honing (,Untersuchungen“, 1911) hat die Keimprozente bei verschiedenen Formen bestimmt und dabei beobachtet, daß die nicht keimenden Samen größtenteils keinen guten Kern hatten. Er gibt die Keimprozente für eine Anzahl Individuen von O. Lamarckiana und jeweils für Gruppen von je 5 Früchten getrennt (pag. 79, Tabelle VI). Unter der noch zu prüfenden Voraussetzung, daß das Verhältnis zwischen gesunden und tauben Samen für jedes Individuum konstant ist, halten wir uns jeweils an die höchste bei einem Individuum gefundene Keimzahl. Diese höchsten Keimprozente sind für 9 Pflanzen: 33, 36, 38, 43, 44, 35, 33, 41, 31. Weil nur die vollentwickelten Samen keimen können und auch diese nicht notwendig alle keimen müssen, dürfen wir annehmen, daß Honing sicher kein Exemplar mit nur 25\% guter Samen, sicher mehrere Individuen mit mehr als $37,5 \%$, also wohl mit 50\% guter Samen, und wahrscheinlich kein Individuum mit mehr als 50\% guter Samen in Händen gehabt hat. Es können also Linien gewesen sein, die teils nach $2: 2$ und teils nach $6: 10$ spalteten.

Unter den tauben Samen lassen sich bei O. Lamarckiana gewöhnlich mindestens zwei Typen wohl unterscheiden, solche mit kleinem, bei schwacher Vergrößerung sichtbarem Embryo und vielzelligem Endosperm, und andere mit winzigen Spuren von Embryo und Endosperm (vgl. oben pag. 134). Diese beiden Typen traten bei einigen Materialien in ziemlich genau gleichen Zahlen auf. Unter 93 tauben Samen der O. Lamarckiana von de Vries waren 45 mit kleinem Embryo und 
48 ohne Embryo, von den zuerst mit der Hand geschnittenen Samen des Münchener Materials waren 20 ohne Embryo und 21 hatten einen knopfförmigen Embryo. Wahrscheinlich entsprechen diese beiden Typen zwei verschiedenen Gruppen von Faktorenkombinationen. Sogar innerhalb der beiden Typen scheinen bei sorgfältiger Beobachtung weitere Unterteilungen möglich. Bei einzelnen Materialien waren die kleinen Embryonen teils kugelig, teils beträchtlich größer und zweilappig; so bei der weißnervigen Linie 72 A; leider ist hier bei einem Teil der tauben Samen die Beschaffenheit des Embryo nicht festgestellt worden. An den Mikrotomschnitten von dem frei bestäubten Münchener Material ist in den ganz schlechten Embryosäcken die Zellenzahl im Embryo und besonders im Endosperm ziemlich verschieden. Vielleicht sind auch hier an reichlichem Material verschiedene Typen in festen Zahlenverhältnissen zu unterscheiden. Unter recht günstigen Umständen könnte man also vielleicht die ausfallenden Kombinationen des Schemas auf pag. 137 mit bestimmten Hemmungsformen des Embryosackes identifizieren. Dazu sind aber Individuen von sorgfältig analysiertem Genotypus nötig.

Genau gleich viel gute und taube Samen haben dann die Mutanten nanella und rubrinervis; sie besitzen also, wie die weißnervigen Lamarckiana-Linie $72 \mathrm{~A}$, nur einen Faktor, der homozygotisch nicht realisiert werden kann. Daß sie in gesunde und taube Samen spalten, stimmt mit dem regelmäßigen Vorkommen der laeta-Spaltung in ihren Kreuzungen überein (de Vries 1913, pag. 195, 218). Wie diese beiden Mutanten verhält sich in den Erzeugung tauber Samen die O. suaveolens Dest., die Herr Prof. de Vries im vergangenen Jahr (1913) im Forst von Fontainebleau gesammelt hat. Von dieser Art, die nach brieflicher Mitteilung von Herrn Prof. de Vries mit O. grandiflora Ait. nicht identisch ist, sind also bei der Kreuzung mit den älteren Arten in $F_{1}$ auch zwei Formen zu erwarten. Daß O. grandiflora bei Kreuzung mit $O$. biennis und muricata Zwillinge gibt, hat Davis (vgl. de Vries 1913, pag. 297) gefunden; das Davis'sche" Material wäre also auch auf taube Samen zu prüfen.

Bei O. gigas, die de Vries als sehr seltene Mutante aus O. Lamarckiana erhalten hat, sind taube Samen seltener als bei O. Lamarckiana; bei $\mathrm{Nr}$. 10 sind $13 \%$, bei Nr. 11 etwa $27 \%$ taube Samen gefunden worden. Der Riesentypus Kombination 7 von Heribert-Nilss on (1912, pag. 132) erweist sich insofern sehr gigas-ähnlich, als er sicher sehr wenig taube Samen bringt, sogar noch weniger als 0 . gigas. In der Linie $22 \mathrm{~A}$ ist kein schlechter Same gefunden worden, in $22 \mathrm{~B}$ sehr wenige. Wie alles das zu 
deuten ist, darüber läßt sich erst reden, wenn die Kreuzungen zwischen O. gigas und den anderen Arten, besonders die mit O. Lamarckiana, auf das Vorkommen tauber Samen geprüft sind.

Wenn O. Lamarckiana mit einer der Mutanten gekreuzt wird, entstehen meistens in $\mathrm{F}_{1}$ Zwillinge. Diese Mutanten geben selber bei Kreuzung mit den älteren Arten Zwillinge, und von nanella und rubrinervis wissen wir schon, daß sie bei Selbstbefruchtung zur Hälfte taube Samen bringen. Sie sind also im laeta-Faktor heterozygotisch wie die Stammart Lamarckiana, und wenn sie mit dieser verbunden werden, ist die Kreuzung in Beziehung auf den laeta-Faktor identisch mit einer Selbstbefruchtung. Es ist demnach zu erwarten, daß bei diesen Kreuzungen die Zygoten mit LL und 11 ausgeschaltet werden, daß also taube Samen zu finden sind. Dasselbe gilt für die Kreuzungen zwischen den Mutanten. Die Zwillinge, die bei all diesen Kreuzungen auftreten (z. B. Lamarckiana und nanella aus Lamarckiana $\times$ nanella oder reziprok, Lamarckiana und rubrobusta aus Lamarckiana $\times$ rubrinervis oder reziprok), haben demnach mit der laeta-velutina-Spaltung nichts zu tun, weil sie jeweils beide im laeta-Faktor heterozygotisch sind. Wir werden unten auf sie zurückkommen.

Die Berücksichtigung etwa auftretender tauber Samen wird in Zukunft bei den Önotheren nicht mehr versäumt werden dürfen. Besonders interessant wird es z. B. sein, zu erfahren, wie O. scintillans sich bei Selbstbefruchtung verhält. Sie zeigt bei Kreuzungen, wie muricata $\times$ scintillans, die Spaltung in laeta und velutina (de Vries 1913, pag. 260), ist also im laeta-Faktor heterozygotisch. Außerdem spaltet sie bei Selbstbefruchtung in Lamarckiana, scintillans und oblonga (pag. 257), ist also noch in mindestens einer weiteren Einheit dauernd heterozygotisch. Weiter liefert die Kreuzung scintillans $\times$ biennis-Chicago neben laeta und velutina auch scintillans (pag. 261). Endlich spaltet scintillans $\times$ biennis in scintillans und eine Bastardrasse, die mit Lamarckiana $\times$ biennis übereinstimmt (pag. 261); diese letztere entspricht, wie auseinandergesetzt wurde, einer velutina, bei der zuletzt genannten Kreuzung wäre also die fehlende laeta in Form yon tauben Samen aufzusuchen. Ebenso ist der Drilling scintillans neben der laeta und der velutina in der Kreuzung muricata $\times$ scintillans zu suchen.

O. biennis $\times$ oblonga gibt in $\mathrm{F}_{1}$ nur velutina, die laeta fehlt. Die Unterdrückung des zweiten Bastardtypus dürfte aber erst nach der Zygotenbildung erfolgen, denn die reziproke Kreuzung hat einmal Zwillinge ergeben (de Vries 1913, pag. 266 und 267). 
Die Mutante brevistylis gibt bei Kreuzung mit O. Lamarckiana eine monotype $\mathrm{F}_{1}$-Generation, die der $\mathrm{O}$. Lamarckiana gleicht und bei Selbstbefruchtung regelrecht mendelt, in drei Viertel Lamarckiana und ein Viertel brevistylis spaltet (de Vries z. B. 1903, pag. 429). Die Zwillingsbildung in $\mathrm{F}_{1}$ scheint also zu fehlen, es bleibt aber zu prüfen, ob nicht taube Samen vorkommen, und wenn ja, ob sie zu $50 \%$ oder zu $75 \%$ auftreten.

Das sind nur ein paar Beispiele aus dem riesigen von de Vries gesammelten Material, in denen von der Aufsuchung der tauben Samen klärende Aufschlüsse zu erwarten sind. Es ist kaum zu bezweifeln, daß die jetzt sehr schwer zu übersehenden, außerordentlich mannigfaltigen Vererbungsverhältnisse in der Gattung Oenothera wesentlich einheitlicher erscheinen werden, wenn man auf die ausgeschalteten Zygoten achtet.

Natürlich könnte es auch zygotische Kombinationen geben, die wohl bis zu fertigen Embryonen sich entwickeln, aber dann nicht zu keimen vermögen. Man sieht ja verhältnismäßig selten alle vollentwickelten Samen von Önotheren keimen. Nach Honing (,,Untersuchungen", pag. 79) ist aber die Keimfähigkeit der Samen für ein Individuum nicht konstant; das Keimprozent pflegt verschieden zu sein je nach dem Ort der Frucht in der Ähre, während wir das Verhältnis zwischen vollausgebildeten und tauben Samen schon jetzt, vor der natürlich notwendigen Prüfung reicheren Materials, mit großer Wahrscheinlichkeit für konstant halten dürfen. Das größte bei einem Individuum gefundene Keimprozent ist deshalb höchstens gleich der Prozentzahl der aus genotypischer Ursache durchaus lebensfähigen Samen, aus Früchten mit geringerer Keimkraft bleibt also eine Anzahl ,genotypisch gesunder" Samen ungekeimt. Diese Unsicherheit der Keimung läßt die Aussicht, Kombinationen aufzufinden, die erst bei der Keimung versagen, sehr gering erscheinen.

Dagegen ist zu erwarten, daß auch in anderen Gattungen das Mißlingen von Kreuzungen gelegentlich in ähnlicher Weise zustande kommt wie bei 0 . muricata-Venedig $\times$ biennis. Baur hat, ohne einen sicheren Fall zu kennen, diese Vermutung schon geäußert (1911, pag. 221). Es gibt da wohl allerhand einfache, lohnende Aufgaben für zytologische Untersuchung.

Bei Gärtner (1849, pag. 93ff.) finden sich in dem Kapitel „,Von der unvollkommenen Bastardbefruchtung" zahlreiche Fälle angegeben, in denen der Same nach der Bestäubung mehr oder weniger weit sich entwickelt, ohne einen keimfähigen Embryo einzuschließen. Die oben 
von Önotheren beschriebenen Erscheinungen entsprechen dem, was Gärtner „,täuschende Befruchtung (Fructificatio erronea)“ nennt (pag. 99): „Die Frucht ist meist normal ausgebildet und enthält neben vielen staubartig vertrockneten Eichen und vielen tauben auch, dem Anschein nach, vollkommene Samen mit einem medullosen Kern, welcher aber keinen Embryo einschließt." Unter den Formen, von denen diese Art der Bastardsamenbildung bekannt ist, nennt Gärtner verschiedene Arten von Oenothera, nämlich: O. Fraseri $\times$ fructicosa, Fraseri $\times$ glauca, fructicosa $\times$ pumila, grandiflora $\times$ nocturna, grandiflora $\times$ rosea (pag. 100). Die Verschiedenheit in der Ausbildung der Samen innerhalb einer und derselben Frucht, von der Gärtner wiederholt (z. B. auch pag. 101) spricht, kann ganz wohl eine Erscheinung fluktuierender Variabilität sein, ist aber vielleicht auch gelegentlich auf Heterozygotie der verwendeten Materialien zurückzuführen. Selten gelingende Kreuzungen, die nur dann und wann einige keimfähige Samen liefern, sind wohl nach dieser Richtung noch zu prüfen.

c) Die Mutabilität der O. Lamarckiana.

Die Mutationen in der Gattung Oenothera beruhen nach de Vries (1913, pag. 15, 281, 333) zur Hauptsache auf einer Zustandsänderung gewisser Faktoren (Pangene). Die Erbeinheiten sind in nicht mutabeln Formen in zwei Lagen oder Zuständen vorhanden, nämlich aktiv oder inaktiv (homozygotisch an- oder abwesend). Bei mutabelen Formen kommt dazu eine dritte Lage, die labile, im Gegensatz zu den beiden stabilen. Im Phänotypus manifestiert sich ein labiler Faktor gerade so wie ein aktiver. Wenn die labile Lage in eine stabile übergeht, so tritt Mutation ein. Die Entstehung der zwergigen O. nanella z. B. aus O. Lamarckiana ist darauf zurückzuführen, daß das in Lamarckiana labile Gen für hohe Statur inaktiv wird. Nanella kann, weil der Staturfaktor stabil geworden ist, in Beziehung auf die Statur nicht mehr mutieren. Bei der Mutation der O. Lamarckiana in rubrinervis wird das labile Gen für Festigkeit des Stengels inaktiv, der Stengel damit spröde, und zugleich wird der Staturfaktor im umgekehrten Sinn wie bei nanella verändert, er wird aktiv, so daß rubrinervis keine Zwerge hervorbringen kann.

Der labile Zustand eines Faktors äußert sich vor allem bei Kreuzungen. Wird O. Lamarckiana z. B. mit nanella verbunden, so treten schon in der ersten Generation Zwerge neben hochstengeligen Pflanzen auf; mitunter sind beide etwa gleich zahlreich. Der labile Faktor scheint also zwischen dem aktiven und dem inaktiven Zustand 
zu schwanken. Das Ergebnis einer solchen „Mutationskreuzung“ ist somit ein ganz anderes als das einer Kreuzung zwischen zwei Formen, die das betreffende Gen alle beide in stabilem Zustand besitzen. Z. B. ist die Kreuzung muricata $\times$ nanella und ebenso rubrinervis $\times$ nanella in $F_{1}$ in der Statur einförmig, Zwerge werden erst in $F_{2}$ (bei Selbstbefruchtung der $\mathrm{F}_{1}$ ) abgespalten. Die Spaltung in der ersten Generation (,alternative Vererbung" nach Gates 1911, pag. 371) ist das wichtigste Kennzeichen labiler Faktoren. Dazu kommt, daß ein labiler Faktor die Spaltbarkeit nur dann zeigen soll, wenn der zur Kreuzung verwendeten Art das betreffende aktive Spaltungsvermögen innewohnt; doch diesen Punkt haben wir schon erledigt (pag. 133).

De Vries weist selbst darauf hin (1913, pag. 283), daß das Verhalten eines labilen Faktors bei Kreuzung mit dem eines heterozygotischen, mendelnden übereinstimmt. Nehmen wir an, O. Lamarckiana sei im Staturfaktor heterozygotisch, nanella sei ein homozygotischer absenceTypus, so muß die erste Generation der Kreuzung zur Hälfte aus hohen Individuen und zur Hälfte aus Zwergen bestehen. Aber der grundlegende Unterschied zwischen einer mutabelen und einer heterozygotischen Form soll in dem Verhalten bei Selbstbefruchtung bestehen: die heterozygotische spaltet neben Heterozygoten Plus- und Minus-Homozygoten ab, die mutable Form dagegen mit ihrem labilen Gen bleibt zur Hauptsache konstant, nur ganz selten springt eine Mutante ab, in der das labile Gen nach der positiven oder nach der negativen Seite stabil geworden ist.

Als labil betrachtet nun de Vries in O. Lamarckiana auch den laeta-Faktor. Denn bei Kreuzung mit den älteren Arten entstehen in der $\mathrm{F}_{1}$-Generation laeta und velutina nebeneinander, während bei Selbstbefruchtung nie Abspaltung von solchen Formen eintritt, welche die laeta- oder die velutina-Eigenschaften rein (stabil, homozygotisch) zur Schau trügen. De Vries identifiziert auch keine seiner Mutanten mit einer solchen reinen laeta-, velutina-, densa- oder laxa-Form. $\mathrm{Er}$ sagt, daß die laeta- und densa-Eigenschaften ,zwar bei Kreuzungen zu unmittelbaren Spaltungen Veranlassung geben, aber, soweit bekannt, bis jetzt noch nicht durch Mutation neue Arten haben auftreten lassen“" (1913, pag. 334).

Von diesem laeta-Faktor glauben wir aber jetzt zu wissen, daß er bei Selbstbestäubung der Formen, die ihn besitzen, zu einfacher Mendel-Spaltung führt; nur weil die beiderlei Homozygoten regelmäßig zugrunde gehen, ist das bisher übersehen worden. Es ist also wahrscheinlich, daß der laeta-Faktor in O. Lamarckiana und ihren 
Mutanten in heterozygotischem Zustand vorhanden ist und nicht etwa in einem irgendwie davon verschiedenen.

Schwieriger als die laeta-Spaltung ist das Auftreten der Mutanten auf Heterozygotie und Mendel-Spaltung zurückzuführen. Daß der Versuch nicht aussichtlos erscheint, hat Heribert-Nilss o n (1912, pag. 193, 200) dargetan. Hier soll nur auf einen Punkt hingewiesen werden. Bei der laeta-Spaltung besteht $\mathrm{z}$ wischen dem Ergebnis der meisten Kreuzungen und dem der Selbstbefruchtung insofern gute Übereinstimmung, als wir auf beiden Wegen Spaltungszahlen erhalten, die für einfache Heterozygotie der O. Lamarckiana in der betreffenden Eigenschaft sprechen. Weniger klar liegen die Verhältnisse z. B. für die Beurteilung der nanellaEigenschaften. Werden O. Lamarckiana und nanella miteinander gekreuzt, so erscheinen in der $\mathrm{F}_{\mathbf{1}}$-Generation im Mittel ungefähr ein Viertel Zwerge (genauer 21\%, vgl. Mutationstheorie II, 411). Das Mutationsprozent an nanella war für die benutzten Lamarckiana-Pflanzen etwa 1\% (ebenda, pag. 408), während man unter der allereinfachsten Annahme, von dem Ergebnis der Kreuzung ausgehend, bei Selbstbefruchtung der O. Lamarckiana etwa $1 / 16$, d. h. ungefähr $6 \%$ Zwerge erwarten möchte. Doch wird sich der scheinbare Widerspruch wohl lösen lassen. Es ist auch nicht zu vergessen, daß die an entwickelten Nachkommen ermittelten Zahlenverhältnisse nur dann ganz zuverlässig sind, wenn sämtliche fertig ausgebildeten Samen keimen. Durch verschiedenes Keimungsvermögen der differenten Formen können die Zahlenverhältnisse ja weit gefälscht werden.

d) Die angebliche Konstanz der Artbastarde.

Zum Schluß müssen wir noch einmal zu dem Ausgangspunkt der Untersuchung zurïckkommen. In keinem der untersuchten Fälle ist Merogonie beobachtet worden, und jetzt ist darauf hinzuweisen, daß die experimentellen Voraussetzungen, auf die Goldschmidt seine Hypothese von der Merogonie gründete, auch gar nicht gegeben sind. Die fraglichen einfachen Bastarde sind nicht konstant, und die doppeltreziproken Bastarde kehren nicht rein zum Typus des einen Elters, des ,peripherischen“, zurück.

Baur hat gegenüber den Angaben von de Vries schon 1911 (pag. 219) hervorgehoben, daß er den Bastard O. muricata $\times$ biennis in $\mathrm{F}_{2}$ nicht einheitlich gefunden hat. Darauf weist er auch bei der Besprechung von Goldschmidt's Merogonie-Arbeit hin (Zeitschr. f. ind. Abstammungslehre 1913, Bd. IX, pag. 136); die beobachteten Unterschiede lagen „vor allem in der Blütengröße und -form". Honing 
(,Doppelnatur“, pag. 234) findet, daß bei O. (biennis $\times$ rubrinervis) laeta die dritte und die vierte Generation untereinander und von der ersten deutlich verschieden waren. In der dritten Generation war ,,von der Mutterpflanze, O. biennis, nichts mehr herauszufinden". Die vierte Generation ,sah nicht wie eine laeta aus, die Blätter waren schmal, sogar schmäler als von O. rubrinervis selbst." Es sind also auch vegetative Charaktere, nicht bloB Blütenmerkmale, in denen die Konstanz fehlt. Auch ,die laeta und velutina aus 0 . muricata $\times$ Lamarckiana sind in erster Generation nicht $\mathrm{zu}$ vergleichen mit denselben in dritter und fünfter Generation" (pag. 235). Ebenso berichtet de Vries in seinem neuen Buch (1913) mehrfach, daß die Bastardrassen in $\mathrm{F}_{2}$ in der Blütengröße mehrförmig sind, wenn sie ihm auch in den vegetativen Organen konstant erscheinen. Z. B. treten bei laeta und velutina in $\mathrm{F}_{2}$,,deutlich zwei Typen auf, deren einer doppelt so große Blüten hat als der andere" (pag. 127).

Auch in den doppeltreziproken Bastarden betrifft die Ausschaltung des ,zentralen“ Elters nicht alle Eigenschaften, nämlich nicht die Größe und Form der Kronblätter. Bei O. (cruciata $\times$ biennis) $\times($ biennis $\times$ cruciata) waren die Petalen bei acht Exemplaren rein linealisch, wie bei der cruciata, bei 22, ,mehr oder weniger verbreitert und verrieten somit den Einfluß von O. biennis deutlich" (de Vries 1913, pag. 98). Bei O. (Hookeri $\times$ biennis $) \times($ biennis $\times$ Hookeri) schwankte die Größe der Blumenblätter zwischen $1,5 \mathrm{~cm}$ und $3 \mathrm{~cm}$ (pag. 99), und zwar bestanden diese Unterschiede zwischen den Individuen, nicht etwa zwischen den Blüten eines und desselben Individuums.

Nach der Entdeckung der tauben Samen bei der Kreuzung O. Lamarckiana $\times$ biennis war mit der Möglichkeit zu rechnen, daß die anscheinende weitgehende Konstanz der selbstbefruchteten Artbastarde durch die Eliminierung gewisser Zygoten zustande kommt. Ich habe mir deshalb außer den schon beschriebenen Materialien auch Samen von solchen Bastardrassen erbeten. Das Ergebnis einiger Zählungen folgt in der Tabelle 2.

Die Bastarde zwischen 0 . biennis und muricata geben also bei Selbstbestäubung $50 \%$ taube Samen. Der Bastard O. Lamarckiana $\times$ biennis hat gar nur 7,6-15\% gute Samen. Wie die in den tauben Samen vorliegenden zygotischen Kombinationen zu deuten sind, kann eingehende hybridologische Analyse vielleicht einmal zeigen. Die gigasFormen scheinen sich wieder abweichend zu verhalten, insofern als die untersuchte Kreuzung sicher sehr wenig taube Samen bringt; unter 27 zum Keimen ausgelegten Samen war kein tauber, unter 185 Samen, 
Tabelle 2.

\begin{tabular}{|c|c|c|c|c|c|c|}
\hline Material & Herkunft & $\begin{array}{c}\text { Gute } \\
\text { Samen }\end{array}$ & $\begin{array}{l}\text { Taube } \\
\text { Samen }\end{array}$ & $\begin{array}{c}\text { Theore- } \\
\text { tische } \\
\text { Zahlen }\end{array}$ & $\begin{array}{c}\text { Mittlerer } \\
\text { Fehler }\end{array}$ & $\begin{array}{l}\text { Spal- } \\
\text { tungs- } \\
\text { ver- } \\
\text { hältnis }\end{array}$ \\
\hline $\begin{array}{l}\text { 1. biennis } \times \text { muricata, } \mathrm{F}_{3} \text {. } \\
\text { 2. biennis } \times \text { muricata, } \mathrm{F}_{4} \text {. } \\
\text { 3. muricata } \times \text { biennis, } \mathrm{F}_{2} \text {. } \\
\text { 4. muricata } \times \text { biennis, } \mathrm{F}_{3} \text {. } \\
\text { 5. Lamarckiana } \times \text { biennis, } \\
\mathrm{F}_{2}, 56 / 8 \times \cdots \text { biennis, } \\
\text { 6. Lamarckiana } \times \text { bien, } \\
\mathrm{F}_{3}, 50 / 25 \times \text {. } . \mathrm{F}_{1}, 24\end{array}$ & $\begin{array}{c}\text { de Vries } \\
" \\
" \\
" \\
\text { Her.-Nilsson } \\
" \\
"\end{array}$ & $\begin{array}{l}48 \\
34 \\
39 \\
38 \\
\\
38 \\
\\
17 \\
27\end{array}$ & $\begin{array}{r}46 \\
26 \\
51 \\
40 \\
\\
217 \\
208 \\
0\end{array}$ & $\begin{array}{l}47: 47 \\
30: 30 \\
45: 45 \\
39: 39\end{array}$ & $\begin{array}{l} \pm 3,87 \\
\pm 4,7\end{array}$ & $\begin{array}{l}2: 2 \\
2: 2 \\
2: 2 \\
2: 2\end{array}$ \\
\hline
\end{tabular}

von denen 9 als verdächtig geöffnet wurden, fand sich ein ganz tauber und ein zweifelhafter Same.

Heribert-Nilsson hält es nach dem ihm vorliegenden Material für möglich (pag. 187), ,daß die von de Vries verfochtene Konstanz (der Oenothera-Bastarde) nur eine Durchschnittskonstanz ist, aus dem Grunde nämlich, weil die Differenzen zwischen den Arten zum größten Teil quantitativer Natur sind, weshalb auch die Spaltung eine graduelle wird“. Wir können jetzt noch weiter gehen. Es gibt nicht einmal eine Durchschnittskonstanz. Der ,konstante“ Bastard O. Lamarckiana $\times$ biennis spaltet ja dauernd qualitativ stark abweichende, nämlich ganz lebensunfähige Kombinationen ab. De Vries selber hat zahlreiche Bastardrassen erzogen, die in einem Teil ihrer Nachkommenschaft immer in zwei oder mehr Typen spalten, wie z. B. O. (biennis-Chicago $\times$ Lamarckiana) laxa, die dauernd atra abspaltet (1913, pag. 151); wie O. (nanella $\times$ Hookeri) laeta, die in laeta und velutina spaltet, und 0 . (nanella $\times$ Hookeri) velutina, die in konstante velutina, in spaltende velutina und in Zwerge spaltet (1913, pag. 228), und dieses Verhalten wird sich wohl als weitverbreitet herausstellen, wenn man bei den konstant erscheinenden Bastarden nach früh ausgeschalteten Zygoten sucht.

Die scheinbar konstanten Bastardrassen zwischen 0. biennis und muricata sind wohl auch nicht bedeutungslos für das Problem der O. Lamarckiana. Sie zeigen, daß durch Verbindung zweier vollkommen fertiler Arten heterozygotische Konstruktionen entstehen können, die unter Zygotenausschaltung dauernd heterozygotisch bleiben. Damit hat die Vermutung, die O. Lamarckiana sei durch Kreuzung hervorgebracht, eine neue Stütze gefunden.

Flora, Bd. 107. 


\section{Zusammenfassung der Ergebnisse.}

1. Bei den untersuchten Artkreuzungen findet normale doppelte Befruchtung statt. Die Chromosomenzahl im Embryo und im Endosperm ist 14. Die untersuchten Verbindungen sind: Oenothera biennis $\times$ muricata, muricata $\times$ biennis, biennis $\times$ Lamarckiana, Lamarckiana $\times$ biennis.

2. Die Kreuzungen 0 . biennis $\times$ muricata und biennis $\times$ Lamarckiana geben lauter gesunde Samen.

3. Die Kreuzung 0 . muricata-Venedig $\times$ biennis liefert kranke Embryonen und Endosperme, die beide sehr früh in der Entwicklung stehen bleiben; die Testa der tauben Samen bildet sich ziemlich normal aus. Das Taubwerden der Samen scheint kein Verhungern, sondern genotypisch bedingt zu sein.

4. Die Kreuzung Lamarckiana $\times$ biennis gibt zur Hälfte gesunde und zur Hälfte taube Samen. Es treten also wie bei der reziproken Kreuzung Zwillingsbastarde auf, von denen aber nur ein Typus, wohl der mit der velutina-Form vergleichbare, sich entwickelt, während der andere, der laeta-Form entsprechende, auf sehr frühem Stadium zugrunde geht.

5. O. Lamarckiana gibt bei Selbstbefruchtung mindestens zur Hälfte taube Samen. Diese stellen die im laeta-velutina-Faktor homozygotischen Kombinationen dar. O. Lamarckiana erhält sich auf diese Weise dauernd in heterozygotischem Zustand und vermag deshalb bei Kreuzung mit 0 . biennis und anderen Arten in der ersten Generation immer zwei Bastardtypen, gewöhnlich laeta und velutina, zu liefern. Die Bildung der Zwillingsbastarde ist demnach eine einfache MendelSpaltung.

6. Die Mutanten O. nanella und rubrinervis erzeugen ebenfalls $50 \%$ taube Samen. Sie sind im laeta-velutina-Faktor dauernd heterozygotisch und geben deshalb bei Kreuzung mit O. biennis usw. Zwillingsbastarde.

7. O. gigas erzeugt weit über $50 \%$ gesunde Samen.

8. Von wildwachsenden Arten bringt O. suaveolens bei freier Bestäubung zur Hälfte gesunde und zur Hälfte taube Samen. O. biennis und O. muricata haben lauter gesunde Samen.

9. Die konstant erscheinenden Bastardrassen 0 . biennis $\times$ muricata, muricata $\times$ biennis, Lamarckiana $\times$ biennis spalten bei Selbstbefruchtung dauernd (mindestens bis zur dritten bzw. vierten Generation) taube Samen $a b$, sind also in Wirklichkeit nicht konstant, vielmehr dauernd spaltende Heterozygoten wie O. Lamarckiana. 
10. Die in den Sätzen $3,4,5,6,9$ ausgesprochene Deutung der tauben Samen wird aufgestellt für den Fall, daß das Verhältnis zwischen tauben und gesunden Samen bei umfangreicheren, schon in die Wege geleiteten Studien an genotypisch einheitlichem Material sich als konstant erweist.

\section{Botanisches Institut. München-Nymphenburg, 28. Februar 1914.}

\section{Zitierte Literatur.}

Baur, Einführung in die experimentelle Vererbungslehre. Berlin 1911.

Gärtner, Versuche und Beobachtungen über die Bastarderzeugung im Pflanzenreich. Stuttgart 1849.

Gates, Studies on the variability and hereditability of pigmentation in Oenothera. Zeitschr. f. indukt. Abstammungs- und Vererbungslehre 1911, Bd. IV, pag. 337.

Geerts, Beiträge zur Kenntnis der Zytologie und der partiellen Sterilität von Oenothera Lamarckiana. Rec. trav. bot. Néerland. 1909, Bd. V, pag. 91.

Goldschmidt, Die Merogonie der Oenothera-Bastarde und die doppeltreziproken Bastarde von de Vries. Archiv f. Zellforschung 1912, Bd. IX, pag. 331.

Heribert-Nilsson, Die Variabilität der Oenothera Lamarckiana und das Problem der Mutation. Zeitschr. f. indukt. Abstammungs- und Vererbungslehre, 1912, Bd. VIII, pag. 89.

Ders., Oenothera-problemet. Svensk bot. Tidskrift 1913, Bd. VII, pag. 1.

Honing, Die Doppelnatur der Oenothera Lamarckiana. Zeitschr. f. indukt. Abstammungs- und Vererbungslehre 1911, Bd. IV, pag. 227.

Ders., Untersuchungen an Oenothera-Früchten und Samen. Rec. trav. bot. Néerland. 1911, Bd. VIII, pag. 65.

Modilewski, Zur Embryobildung von einigen Onagraceen. Ber. d. Deutschen Botanischen Gesellschaft 1909, Bd. XXVII, pag. 287.

Strasburger, Zeitpunkt der Bestimmung des Geschlechtes, Apogamie, Parthenogenesis und Reduktionsteilung. Histolog. Beiträge, Heft 7. Jena 1909.

Tischler, Über die Entwicklung der Samenanlagen in parthenokarpen Angiospermenfrüchten. Jahrb. f. wiss. Bot. 1913, Bd. LII, pag. 1.

De Vries, Die Mutationstheorie, Bd. I 1901, Bd. II 1903. Leipzig.

Ders., Über doppeltreziproke Bastarde von Oenothera biennis L. und O. muricata L. Biolog. Zentralbl. 1911, Bd. XXXI, pag. 97.

Ders., Gruppenweise Artbildung. Berlin 1913. 
O. Renner, Befruchtung u. Embryobildung bei Oenothera Lamarckiana usw.

\section{Figurenerklärung zu Tafel XII und XIII.}

Die Figuren sind mit Zeiss-Apochromat $2 \mathrm{~mm}$ und Komp.-Okular 18 gezeichnet.

Fig. 1 und 2. O. biennis $\times$ muricata-Süddeutschland. 1 Metaphase, 2 Prophase aus einem älteren Embryo.

Fig. 3-10. O. biennis $\times$ muricata-Venedig.

Fig. 3. Befruchtetes Ei in früher Prophase.

Fig. 4. Ebenso in später Prophase.

Fig. 5. Metaphase im Ei, schräg durchschnitten; nicht etwa Anaphase.

Fig. 6. Zweite Mitose im Embryo, Metaphase.

Fig. 7. Spindel im befruchteten Ei, Metaphase.

Fig. 8. Zweite Mitose im Embryo, Prophase; nach einem nicht durchschnittenen Kern gezeichnet.

Fig. 9. Zweikerniger Embryo. Kerne „,in Degeneration“.

Fig. 10. Zweizelliger Embryo. Kerne ebenso, mit sehr unregelmäßig geformten Nukleolen.

Fig. 11. O. biennis $\times$ muricata-Süddeutschland. Schlecht fixierte Prophase aus einem etwa 20 zelligen Embryo.

Fig. 12-16. Endosperm von 0 . biennis $\times$ muricata.

Fig. 12. Metaphase,

Fig. 13. Anaphase aus älterem Endosperm.

Fig. 14. Prophase aus sehr jungem Endosperm.

Fig. 15 und 16. Metaphasen aus sehr jungem Endosperm.

Fig. 17-23. 0. biennis $\times$ Lamarckiana.

Fig. 17. Prophase im Suspensor.

Fig. 18. Prophase aus einem mehrzelligen Embryo.

Fig. 19. Anaphase aus einem mehrzelligen Embryo.

Fig. 20. Metaphase der zweiten Teilung, im Suspensor.

Fig. 21. Metaphase aus sehr jungem Endosperm.

Fig. 22 und 23. Metaphasen aus mehrzelligen Embryonen.

Fig. 24. O. Lamarckiana $\times$ biennis. Späte Anaphase aus einem mehrzelligen gesunden Embryo. 


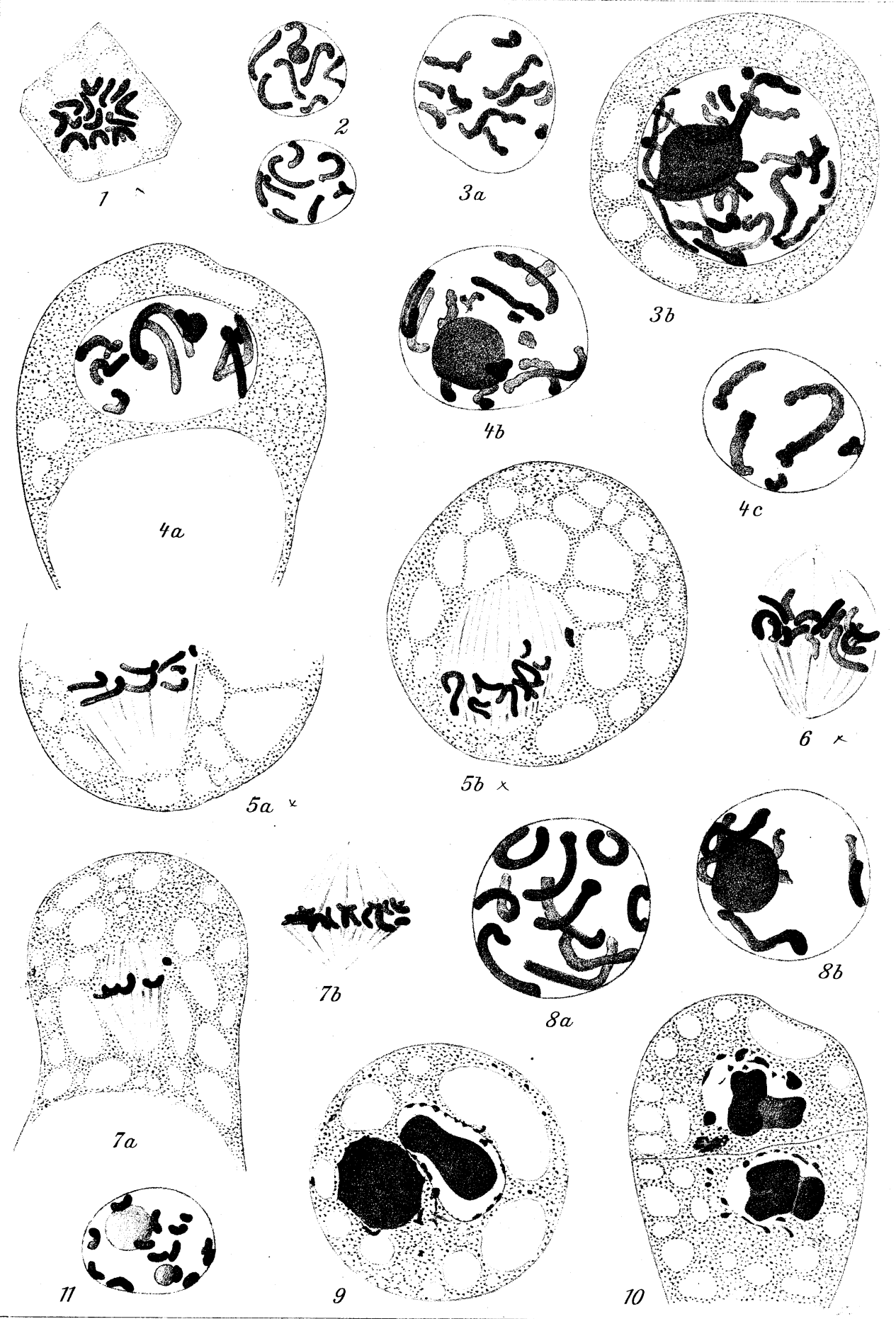



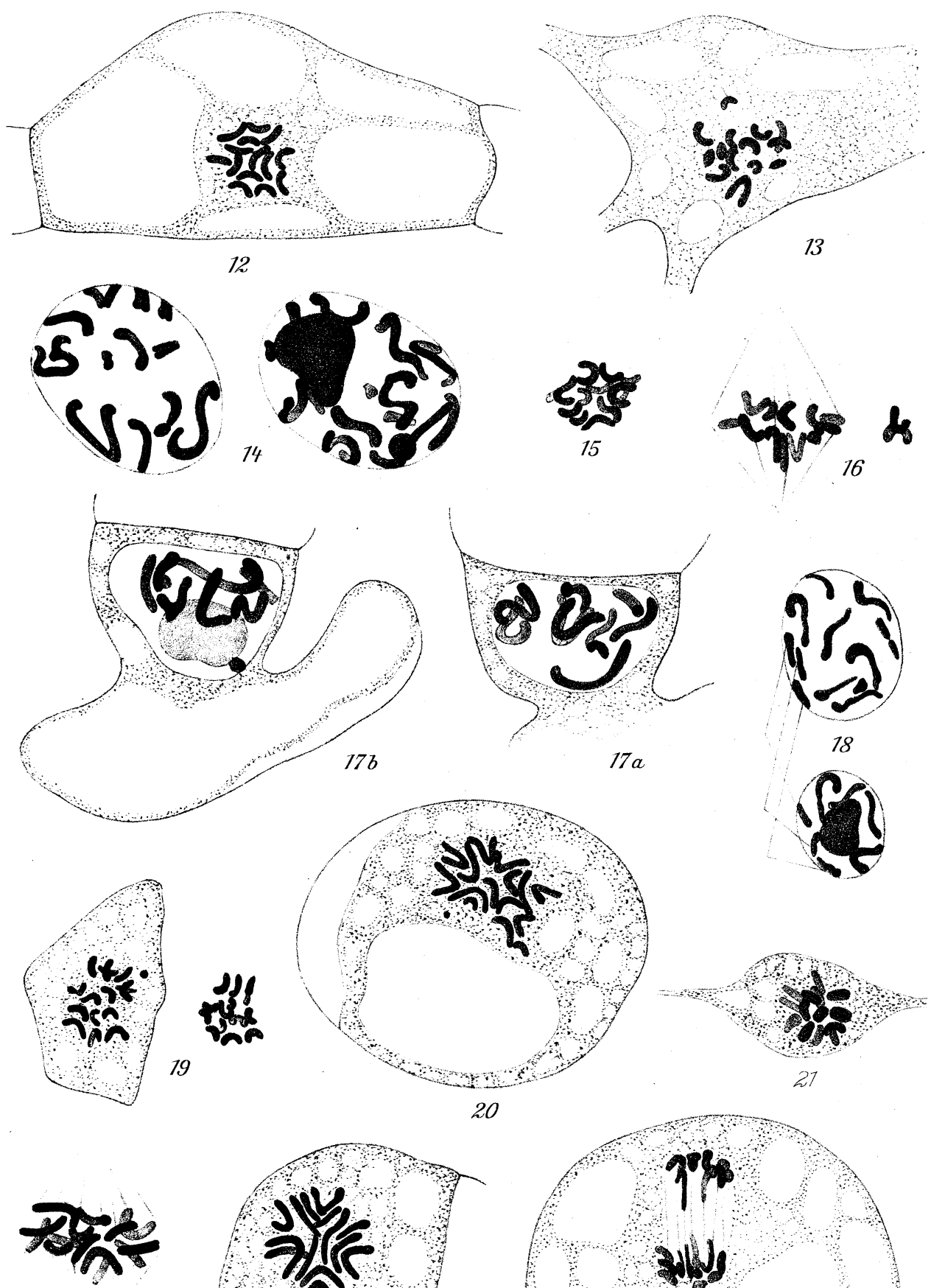

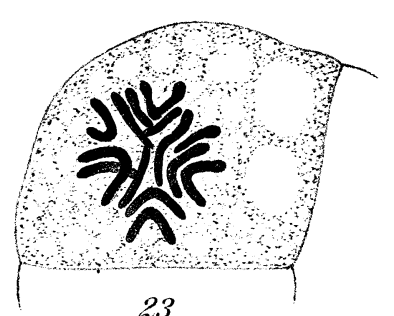

23

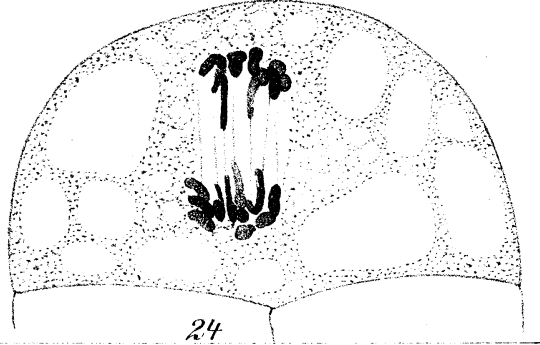

